\title{
SCIENTIFIC REPORTS

\section{Genomic profiling of antimicrobial resistance genes in clinical isolates of Salmonella Typhi from patients infected with Typhoid fever in India}

\author{
Amit Katiyar ${ }^{1,2,4}$, Priyanka Sharma ${ }^{1,4}$, Sushila Dahiya ${ }^{3}$, Harpreet Singh ${ }^{2}$, Arti Kapil ${ }^{3 凶} \&$ \\ Punit Kaur ${ }^{1 凶}$
}

The development of multidrug resistance in Salmonella enterica serovar Typhi currently forms a major roadblock for the treatment of enteric fever. This poses a major health problem in endemic regions and extends to travellers returning from developing countries. The appearance of fluoroquinolone non-susceptible strains has resulted in use of ceftriaxone as drug of choice with azithromycin being recommended for uncomplicated cases of typhoid fever. A recent sporadic instance of decreased susceptibility to the latest drug regime has necessitated a detailed analysis of antimicrobial resistance genes and possible relationships with their phenotypes to facilitate selection of future treatment regimes. Whole genome sequencing (WGS) was conducted for 133 clinical isolates from typhoid patients. Sequence output files were processed for pan-genome analysis and prediction of antimicrobial resistance genes. The WGS analyses disclosed the existence of fluoroquinolone resistance conferring mutations in gyrA, gyrB, parC and parE genes of all strains. Acquired resistance determining mechanisms observed included catA1 genes for chloramphenicol resistance, dfrA7, dfrA15, sul1 and sul2 for trimethoprim-sulfamethoxazole and $b l a_{T E M-116} / b l a_{T E M-1 B}$ genes for amoxicillin. No resistance determinants were found for ceftriaxone and cefixime. The genotypes were further correlated with their respective phenotypes for chloramphenicol, ampicillin, co-trimoxazole, ciprofloxacin and ceftriaxone. A high correlation was observed between genotypes and phenotypes in isolates of $S$. Typhi. The pangenome analysis revealed that core genes were enriched in metabolic functions and accessory genes were majorly implicated in pathogenesis and antimicrobial resistance. The pan-genome of $S$. Typhi appears to be closed $\left(B_{p a n}=0.09\right.$ ) as analysed by Heap's law. Simpson's diversity index of 0.51 showed a lower level of genetic diversity among isolates of $S$. Typhi. Overall, this study augments the present knowledge that WGS can help predict resistance genotypes and eventual correlation with phenotypes, enabling the chance to spot AMR determinants for fast diagnosis and prioritize antibiotic use directly from sequence.

Typhoid fever, a multisystemic disease related to Salmonella enterica serovar Typhi (S. Typhi) infection is a global threat due to increasing antibiotic resistance to antityphoidal agents in practice ${ }^{1,2}$. Antimicrobial-non-susceptible Salmonella infections not only increase disease severity, but also enhance cost of antibiotic treatment and need for hospitalization resulting in economic losses ${ }^{3,4}$. Resistance in typhoidal salmonellae surfaced majorly after introduction of therapy with chloramphenicol ${ }^{5}$. The emergence of multiple drug resistant isolates collectively resistant to chloramphenicol, ampicillin and co-trimoxazole made ciprofloxacin the drug of choice to treat enteric fever. Wide overuse of this drug generated non-susceptible strains due to appearance of mutations in the target enzyme, DNA gyrase and topoisomerase $\mathrm{IV}^{6,7}$. Discontinuation of older drug regime removed mutational pressure resulting in reappearance of chloramphenicol susceptible strains. However, the possible generation of

${ }^{1}$ Department of Biophysics, All India Institute of Medical Sciences, New Delhi, 110029, India. ${ }^{2}$ ICMR-AlIMS Computational Genomics Center, Division of I.S.R.M., Indian Council of Medical Research, New Delhi, 110029, India. ${ }^{3}$ Department of Microbiology, All India Institute of Medical Sciences, New Delhi, 110029, India. ${ }^{4}$ These authors contributed equally: Amit Katiyar and Priyanka Sharma. ${ }^{\circledR e}$-mail: akapilmicro@gmail.com; punitkaur1@hotmail. com 
plasmid mediated antimicrobial resistance determinants by horizontal transfer has prevented its reuse in clinical practice ${ }^{8}$. Sporadic episodes of extended spectrum beta lactamase genes conferring resistance to current third generation cephalosporins have also emerged ${ }^{9,10}$. Recent emergence of $S$. Typhi isolates resistant to azithromycin in Bangladesh indicated an increasing trend in resistance to azithromycin as compared to the rest of the world ${ }^{11}$. Of great concern is a recent study from Pakistan that reports the outbreak of enteric fever caused by extremely drug resistant (XDR) strains of $S$. Typhi ${ }^{12}$. In this scenario of evolving resistance mechanisms, an examination of the basis of resistance to existing drugs coupled with a genotypic-phenotypic analysis can aid in devising alternative therapeutic targets or strategies for the treatment of multidrug resistant bacterial infections.

Advancements in next generation sequencing technologies have aided whole genome sequencing (WGS) and evaluation of the complete DNA sequence of a bacterium, making it an ideal technique for surveillance ${ }^{13}$. WGS provides definitive genotype information and gives best possible resolution for characterization of an individual organism. Furthermore, strains possessing identical resistance phenotypes conferred by different mechanisms can also be differentiated ${ }^{14}$. Antimicrobial resistance determination by WGS can complement traditional laboratory-based surveillance and provide direct insights into their evolution and transmission from one strain to another. Current genome sequencing methods afford improved and exhaustive data related to the pathogen genotypic characteristics together with the identification of virulence determinants, antimicrobial resistance genes and serotypes whereas conventional antimicrobial susceptibility tests yield the phenotypes of strains. WGS data can help in revealing the antibiotic resistance mechanism ${ }^{15-18}$ for drugs not being tested routinely or where the mechanisms of antimicrobial resistance are not yet identified. Several studies have established a strong antimicrobial genotypes-phenotypes correlation in E. coli, Campylobacter and non-typhoidal Salmonella respectively ${ }^{16,19,20}$.

Antimicrobial resistance is either caused by mutations in chromosomal genes (intrinsic resistance) or by acquisition of plasmid mediated resistance determinants (extrinsic resistance). The intrinsic resistance is mainly due to selection pressure whereas extrinsic genes are acquired by horizontal transfer ${ }^{21}$. The recent trends in the development of antimicrobial resistance (AMR) among S. Typhi in India proposed that multidrug resistant (MDR) enteric fever was decreasing in India and being replaced by enteric fever with fluoroquinolone resistant strains $^{22}$. Similarly, hospital-based genomic surveillance for enteric fever in Bengaluru, India using WGS method suggested that large number of isolates showed non-susceptibility to fluoroquinolones ${ }^{23}$. Genome characterization assessment for AMR and pan genome of clinical isolates in endemic countries like India will assist recognition of variations in resistance mechanism and epidemiology leading to better selection of antimicrobial therapy and implementation of appropriate preventive measures. Though typhoidal fever is common across India and specifically in the northern sub-continent, the diversity between the strains is yet unexplored.

In the present study, 133 strains of $S$. Typhi isolated from patients presented with enteric fever to AIIMS hospital during past 24 years were subjected to whole genome sequencing. The pan genome was constructed and the occurrence of plasmid mediated antimicrobial resistance conferring genes and gene mutations determining resistance to anti-typhoidal agents analysed. This was further compared to the phenotypes of each respective strain. The pan genome analysis indicated that the genome remains almost closed. The genetic diversity among $S$. Typhi strains were investigated using the available online resources, such as BacWGSTdb ${ }^{24}$, Center for Genomic Pathogen Surveillance, and Centre for Genomic Epidemiology. These tools offer bacterial typing, rapid classification, source tracing, and phylogenetic relatedness linked to antibiotic resistance genes and clinical data important in a globalized community.

\section{Result and Discussion}

Salmonella draft genome. A total of 13,645 contigs varying from 48 to 654 with an average of 102.59 contigs per genome were generated. The average genome size of $4.6 \mathrm{Mb}$ with reads per genome of $47,78,163$ base pairs corroborates with other Salmonella strains ${ }^{25}$. Likewise, observed averaged G $+\mathrm{C}$ content of $51.97 \%$ corresponded to other isolates ${ }^{26}$. Functional annotation of the genome predicted a total of 4,607 CDSs, 73 tRNAs, and 1 transfer-messenger RNA. Analysis by Kruskal-Wallis statistical test did not yield any significant differences $(\mathrm{P} \leq 0.05)$ amongst the strains related to their CDS, genome size, GC content, and average gene number. The submission detail for isolates, including statistics of genome assembly and transcript annotation is summarized in Table S1 in Supplementary File 1.

Salmonella pan- and core- genome. The pan-genome furnishes a comparative analysis across same species but different strains to determine specific features of that species. The constructed pan-genome offers insights into shared and diverse roles of genes amongst studied isolates. BPGA pipeline with similarity threshold of $90 \%$ identified a total of $4185(70.79 \%)$ non-redundant core genes present in all genomes (Fig. S1, Supplementary File 2) and 1273 (21.53\%) non-redundant accessory genes occurring in at least one but not present in all genomes. Nearly 454 (7.68\%) strain specific unique genes (singletons) with no orthologs in corresponding genomic strains were observed. Overall, the pan-genome with 5912 genes, contains 1.28 times more gene content than each individual Salmonella strain. The core to pan-genome ratio disclosed a relatively high degree of conservation with the core-genome comprising $70.79 \%$ of the pan-genome. The contribution of 1432 new gene families to the pan-genome from 91 genomes implies that it contained on an average 9.22 new genes per genome. The estimated core-genome size (4185 genes) was higher than previously reported core-genomes of $S$. Typhi (3944 genes) ${ }^{27}$ and $S$. Typhimurium $(3846,3910 \text { and } 3890 \text { genes })^{28-30}$. The predicted core genes from this study were found to be ranging from 4016 to 4545 as determined by various pan-genome tools using $S$. Typhi data ${ }^{31}$. Addition of 42 genomes did not supplement the core genome indicating that further augmentation of genomes would not significantly decrease the size of core genome. Genome-wise statistics revealed a wide variation in the shared accessory genes from 129 (PGS-33) to 523 (PGS-26). The singletons were exclusively present in 76 isolates (57.14\%) with the highest in PGS-123 (48 genes). Likewise, 63 genes were exclusively absent in 27 isolates (20.30\%) with a maximum of 11 in PGS-65. The presence or absence of gene-families might be attributed to specific roles in emergence 
of virulence and adaptation to their respective habitats. The pan-genome size and distribution is summarized in Table S2 in Supplementary File 1.

The closed or open nature of pan-genome evaluates the versatility of the studied strains. The expected gene number in the pan and core-genome was calculated via curve fitting in accordance with Heaps' law ${ }^{32}, \mathrm{Y}_{\text {pan }}=\mathrm{A}_{\mathrm{pan}}$. $\mathrm{x}^{\mathrm{B}}{ }_{\mathrm{pan}}+\mathrm{C}_{\mathrm{pan}}$, where $\mathrm{y}$ is the size of pan-genome, $\mathrm{x}$ is the number of genomes and $A_{p a n}, B_{p a n}$, and $C_{p a n}$ the fitting parameters and exponential equations $\left(\mathrm{Y}_{\text {core }}=\mathrm{A}_{\text {core. e- }} \mathrm{B}_{\text {core }} \mathrm{X}+\mathrm{C}_{\text {core }}\right)$, respectively. In this equation, $\mathrm{B}_{\text {pan }}$ defines whether the pan-genome is closed $\left(\mathrm{B}_{\mathrm{pan}}<0\right.$ or $\left.\mathrm{B}_{\mathrm{pan}}>1\right)$ or open $\left(0<\mathrm{B}_{\mathrm{pan}}<1\right)^{33}$. Total number of gene families (pan-genome) and shared gene families (core genome) are plotted for a given number of genomes added sequentially. The plot indicated a minor expansion of the pan-genome (Bpan $=0.09)$ while the core genome appeared to extend to almost a closed state as the addition of newer genomes failed to increment any novel gene in the existing genome (Fig. 1). This supports earlier studies demonstrating $S$. Typhi to be a closed pan-genome ${ }^{34}$.

Functional distribution of genes in pan-genome. The pan-genome was further gauged for functional role of the constituent genes. The core, accessory and unique genes were examined for their diverse features by exploring the functional databases $\mathrm{COG}$ and $\mathrm{KEGG}^{35-38}$. This annotation reflected that the genes are scattered in an assorted range of diverse functional categories throughout the genome. The COG distribution (Fig. 2) predicted the core genome $(41.82 \%)$ to be predominantly associated with metabolic functions like transport and metabolism of nucleotides, amino acids, coenzyme, carbohydrates, secondary metabolites biosynthesis, lipids and inorganic ions, energy production and conversion and transport and catabolism ${ }^{39,40}$. These categories had some representation in the accessory genome. Some genes were implicated in translation and ribosomal structure mechanism. This analysis suggests that the majority of the core genes are essential for cell survival and necessary for the basic activity of the species. Nearly one-third genes in the accessory (33.86\%) and unique (33.31\%) genome were primarily involved in basic cellular processes of 'information storage and processing', namely translation, ribosomal structure and biogenesis, replication, recombination, repair, and transcription. Though observed in the core genome, these genes were highly represented in the accessory genome signifying inter-species variations. The 'cellular processes and signalling' category was enriched among the core genes $(21.65 \%)$ and depleted in the unique (13.32\%) genome. More than one-fifth genes in the core (20.64\%), accessory (24.10\%) and unique $(24.65 \%)$ genome were either poorly categorized or uncategorized (Fig. 3). The COG functional distribution of pan-genome is summarized in Table S3 in Supplementary File 1.

The KEGG functional distribution revealed similar overall division of core genes associated with "metabolisms" that accounted for a huge part in core (61.32\%) compared to accessory (24.59\%), and unique (13.50\%) genomes (Fig. 3). Genes were mainly associated with 'carbohydrate metabolism,' 'overview', 'amino acid metabolism' and 'energy metabolism'. A sizeable gene proportion in unique (88\%) and accessory (50.55\%) genome, compared to the core-genome, participated in 'human diseases' related to 'infectious diseases', 'immune diseases', 'cancer' and 'cardiovascular disease.' Remarkably, 70 genes in the core-genome were associated with pathway 'drug resistances: antimicrobial' comprising 38 ko01503: cationic antimicrobial peptide (CAMP) resistance genes, 23 ko01501: beta-lactam resistance genes, and 9 ko01502: vancomycin resistance genes. This suggests that antibiotic resistance genotype plays a vital role in determining S. Typhi susceptibility. Overall 2711 (64.78\%), 490 (38.49\%), and 413 (90.97\%) genes in core, accessory and unique-genome, respectively were assigned to a KEGG category. The KEGG functional distribution of pan-genome is summarized in Table S4 in Supplementary File 1.

Genotypic and Phenotypic resistance to antityphoidal agents. Antimicrobial susceptibility patterns were detected by WGS for resistance determining genes (genotype) and disk diffusion method (phenotype) for first line antibiotics (co-trimoxazole, amoxicillin, and chloramphenicol), fluoroquinolones (ciprofloxacin, and pefloxacin), third generation cephalosporins (cefixime and ceftriaxone) and macrolides (azithromycin) as per CLSI guidelines. All the strains which were collectively resistant to ampicillin, chloramphenicol and co-trimoxazole were considered as MDR (Multiple drug resistant) strains. The relationships between resistance gene content identified from WGS was deciphered with the drug resistance profile for each corresponding clinical isolate (phenotype). None of the 133 strains tested by phenotypic method was found to be susceptible to all antimicrobial agents. Out of the 133 strains, 111 (83.5\%; 95\% CI, 76.2\% to 88.8\%) revealed non-susceptibility to one antibiotic and 23 (17.3\%; $95 \%$ CI, $11.8 \%$ to $24.6 \%)$ showed non-susceptibility to two or more antibiotics. The antibiotic susceptibility patterns are discussed individually.

Amoxicillin resistance. The amoxicillin resistance is associated with the presence of beta-lactam genes which were observed in $15.79 \%(21 / 133)$ strains by WGS. The most common beta-lactam resistance gene $b l_{T E M-1 B}$ was observed in 19 strains, TEM-1 in 16 strains and $b l a_{T E M 116}$ in one strain (Table 1; Table S5 in Supplementary File 1). The resistance genes encode for the predominant plasmid-mediated $\beta$-lactamases of Enterobacteriacea ${ }^{41}$. Overall, antimicrobial resistance was observed in $12.03 \%$ (16/133) isolates by phenotypic method. Earlier reports for amoxicillin resistance in Salmonella strains isolated pan-India was $3 \%^{4}$. The sensitivity and specificity was $100 \%$ and $95.73 \%$, respectively for beta-lactams (Fig. 4; Table 3; Table S7 in Supplementary File 1).

Chloramphenicol resistance. Chloramphenicol resistance determinants were observed in $15.79 \%(21 / 133)$ strains by WGS (Table 1; Table S5 in Supplementary File 1). Nineteen non-susceptible strains harboured catA1 gene which encodes chloramphenicol acetyltransferase enzyme causing chloramphenicol resistance by chemical modification of the drug molecule, whereas sixteen isolates harboured the catI genes. Our findings are consistent with other studies reporting chloramphenicol susceptibility in S. enterica ${ }^{4,32}$. Antimicrobial resistance to chloramphenicol was $8.27 \%(11 / 133)$ by disk diffusion method. Similar findings have been reported by other studies where resistance gene carriage rate was higher than phenotypically reported resistance ${ }^{42,43}$. The sensitivity was $100 \%$ and specificity was $91.80 \%$ for phenicol resistance (Fig. 4; Table 3; Table S7 in Supplementary File 1). 


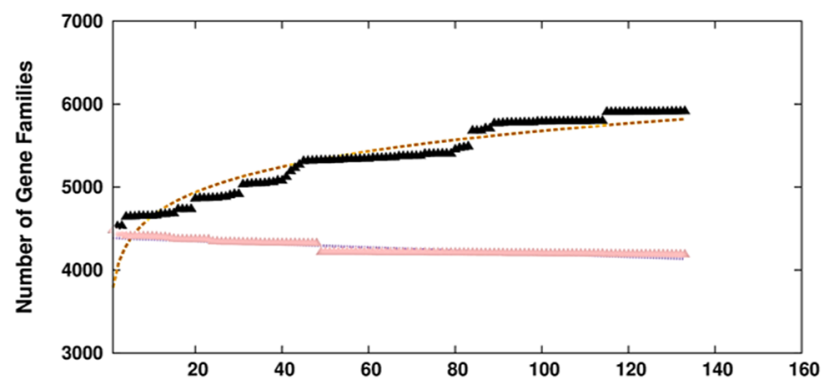

a)

Number of Genomes
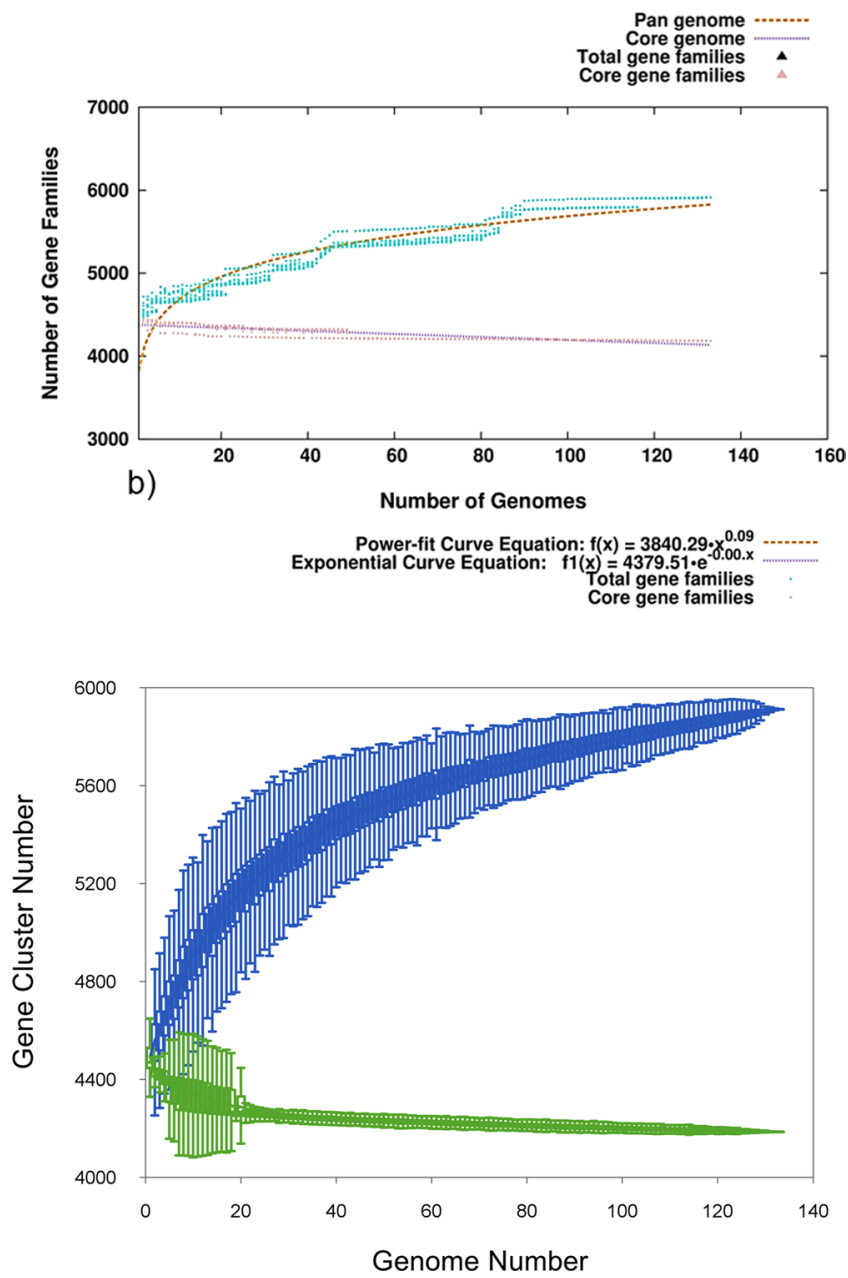

Figure 1. The core-pan genome curve of studied $S$. Typhi genomes. (a) Total number of gene families (pangenome) and shared gene families (core genome) are plotted for a given number of genomes added sequentially. Total gene families are indicated as mustard dashed line while pink dashed line denotes core gene families. (b) The mustard dashed line indicates the least-square fit to the power law function $\mathrm{f}(\mathrm{x})=\mathrm{a} \cdot \mathrm{x}^{\wedge} \mathrm{b}$ where $\mathrm{a}=3840.29$, $\mathrm{b}=0.0852733$. The pink dashed line is the least-squares fit to the exponential decay function $\mathrm{f} 1(\mathrm{x})=\mathrm{c} \cdot \mathrm{e}^{\wedge}(\mathrm{d} . \mathrm{x})$ where $\mathrm{c}=4379.51, \mathrm{~d}=-0.000429421$. (c) Pan- and core-genome curve of $S$. Typhi strains disclosed a minor increase in the size of the pan-genome while the core genome appears to almost close.

Co-trimoxazole resistance. Trimethoprim-sulfamethoxazole were considered in combination for treatment as the first-line drug, co-trimoxazole. Out of 133 strains, trimethoprim resistance determining genes were found in $15 \%$ isolates (20/133). The most prevalent $d f r A 7$ was observed in 18 isolates followed by $d f r A 15$ and $d f r A 1$ in three isolates. Likewise, gene sull and sul2, encoding dihydropteroate synthases known to disseminate sulfamethoxazole resistance, were detected in overall $15.79 \%$ isolates $(21 / 133)$, where 16 isolates harboured both sul1 and sul 2 genes and separately sul1 and sul2 genes alone were observed in 3 and 2 strains, respectively (Table 1; Table S5 in Supplementary File 1). Overall, antimicrobial resistance to co-trimoxazole was detected in $21(15.79 \%)$ strains by WGS as compared to $12(9.02 \%)$ by phenotypic method. The trimethoprim and sulfamethoxazole resistance markers individually were perceived in two isolates. These results are in concordance 


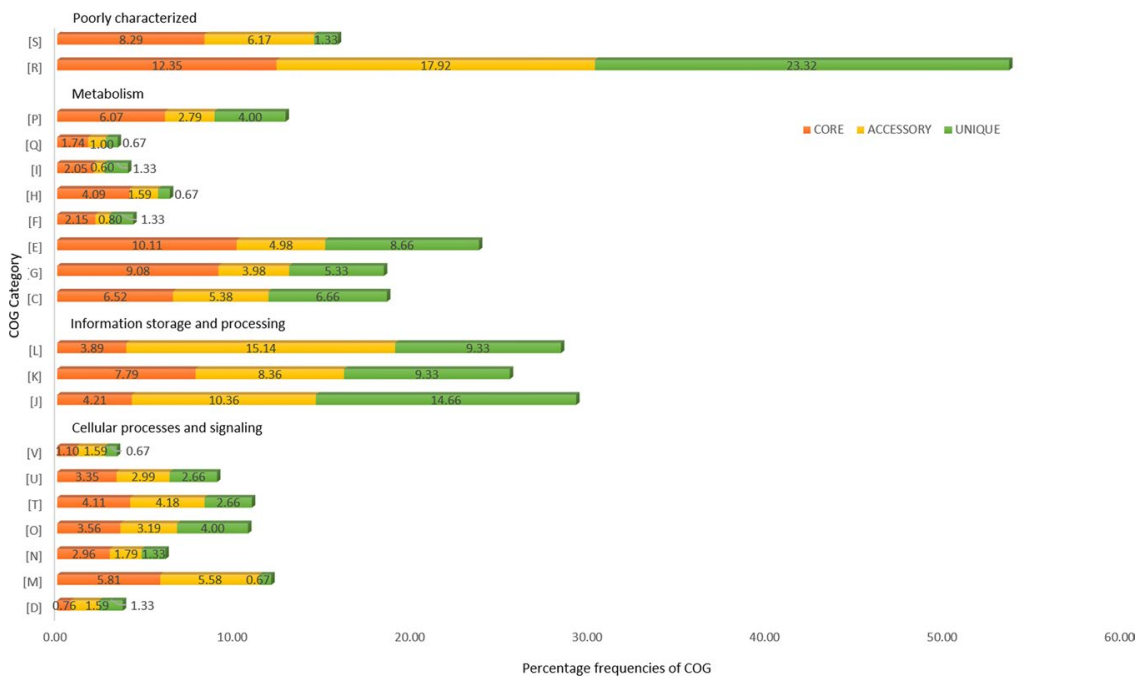

Figure 2. COG functional distribution of pan-genome. The histogram illustrates the predicted functionality of proteins assigned to core (orange), accessory (yellow) and unique (green) genes. The COG families were broadly grouped into four category namely information storage and processing (assigned to the $\mathrm{L}, \mathrm{K}$, and J categories), cellular processes and signalling (assigned to the $\mathrm{V}, \mathrm{U}, \mathrm{T}, \mathrm{O}, \mathrm{N}, \mathrm{M}$ and $\mathrm{D}$ categories), metabolism (assigned to the P, Q, I, H, F, E, G, and C categories), and poorly characterized (assigned to the S, and R categories). COG categories: [C] Energy production \& conversion; [D] Cell cycle control, cell division, chromosome partitioning; [E] Amino acid transport \& metabolism; [F] Nucleotide transport; [G] Carbohydrate transport \& metabolism; \& metabolism; [H] Coenzyme transport \& metabolism; [I] Lipid transport \& metabolism; [J] Translation, ribosomal structure \& biogenesis; [K] Transcription; [L] Replication, recombination \& repair; [M] Cell wall/ membrane/envelope biogenesis; [N] Cell motility; [O] Post-translational modification, protein turnover \& chaperones; [P] Inorganic ion transport \& metabolism; [Q] Secondary metabolites biosynthesis, transport \& catabolism; [R] General function prediction only; [S] Function unknown; [T] Signal transduction mechanisms; [U] Intracellular trafficking, secretion \& vesicular transport; [V] Defense mechanisms.

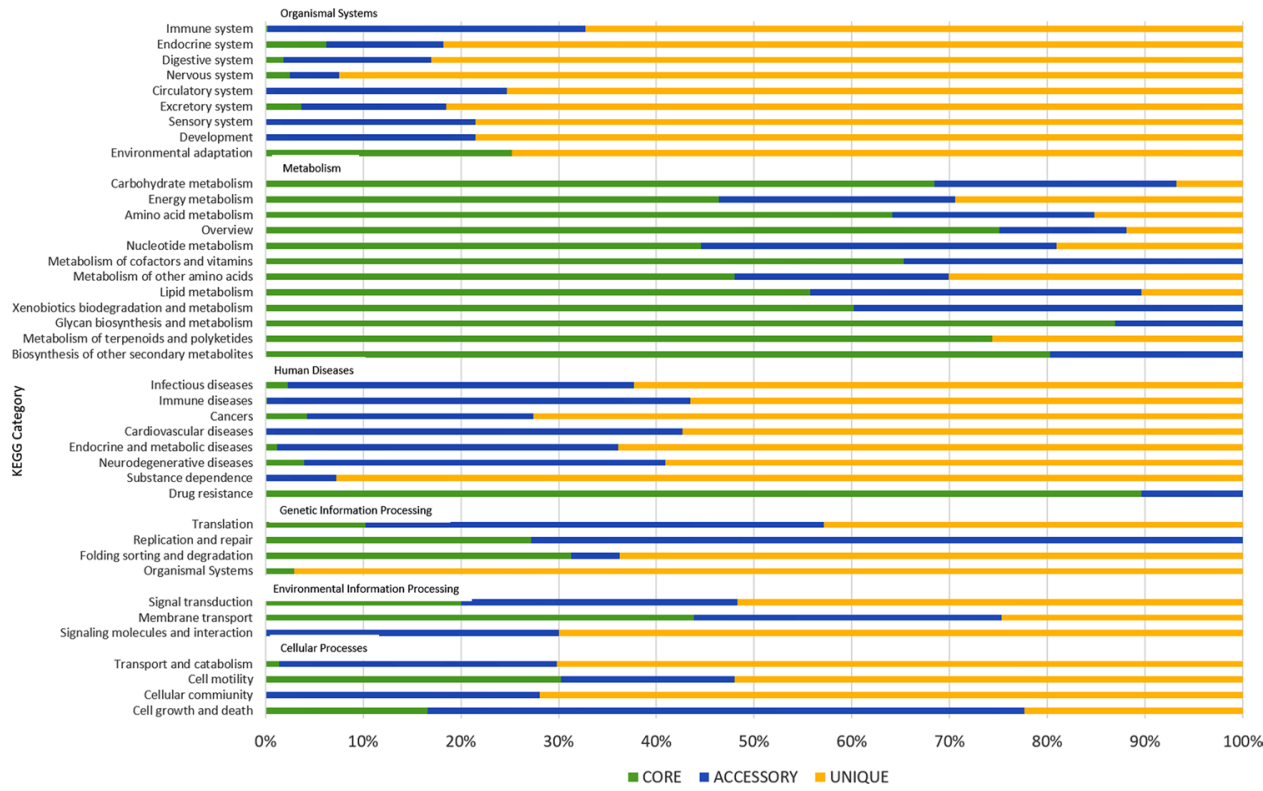

Figure 3. KEGG functional distribution of pan-genome. The graph represent the KEGG functionality of proteins assigned to core (green), accessory (blue) and unique (yellow) genes. The KEGG families were broadly grouped into five category namely cellular processes, human diseases, genetic information processing, environmental information processing, metabolism, and organismal systems.

with previous findings from India which reported antimicrobial resistance in $6 \%$ and $5 \%$ of typhoidal salmonella isolates $^{4,32}$. The sensitivity was $100 \%$ and specificity was $99.56 \%$ for co-trimoxazole (Fig. 4; Table 3; Table S7 in Supplementary File 1). 


\begin{tabular}{|c|c|c|c|c|c|c|c|c|c|c|c|c|c|}
\hline Isolate & AMP & AMX & CHL & CIP & PEF & NAL & SMX & TMP & SXT & TCY & AZM & CFM & CTR \\
\hline PGS-1 & 0 & 0 & 0 & 0 & 0 & 0 & 0 & 1 & 0 & 0 & 0 & 0 & 0 \\
\hline PGS-2 & 1 & 1 & 1 & 1 & 1 & 1 & 1 & 1 & 1 & 1 & 0 & 0 & 0 \\
\hline PGS-3 & 0 & 0 & 0 & 1 & 1 & 1 & 0 & 0 & 0 & 0 & 0 & 0 & 0 \\
\hline PGS-4 & 1 & 1 & 1 & 1 & 1 & 1 & 1 & 1 & 1 & 1 & 0 & 0 & 0 \\
\hline PGS-5 & 0 & 1 & 1 & 1 & 1 & 1 & 0 & 0 & 0 & 1 & 0 & 0 & 0 \\
\hline PGS-6 & 0 & 1 & 1 & 0 & 0 & 0 & 0 & 0 & 0 & 0 & 0 & 0 & 0 \\
\hline PGS-7 & 0 & 0 & 1 & 1 & 1 & 1 & 0 & 0 & 0 & 0 & 0 & 0 & 0 \\
\hline PGS-8 & 1 & 1 & 1 & 1 & 1 & 1 & 1 & 1 & 1 & 1 & 0 & 0 & 0 \\
\hline PGS-9 & 0 & 0 & 0 & 1 & 1 & 1 & 0 & 0 & 0 & 0 & 0 & 0 & 0 \\
\hline PGS-10 & 0 & 0 & 0 & 1 & 1 & 1 & 0 & 0 & 0 & 0 & 0 & 0 & 0 \\
\hline PGS-11 & 0 & 0 & 0 & 1 & 1 & 1 & 0 & 0 & 0 & 0 & 0 & 0 & 0 \\
\hline PGS-12 & 0 & 0 & 0 & 1 & 1 & 1 & 0 & 0 & 0 & 0 & 0 & 0 & 0 \\
\hline PGS-13 & 0 & 0 & 0 & 0 & 0 & 0 & 0 & 0 & 0 & 0 & 0 & 0 & 0 \\
\hline PGS-14 & 0 & 0 & 0 & 1 & 1 & 1 & 0 & 0 & 0 & 0 & 0 & 0 & 0 \\
\hline PGS-15 & 0 & 0 & 0 & 1 & 1 & 1 & 0 & 0 & 0 & 0 & 0 & 0 & 0 \\
\hline PGS-16 & 0 & 0 & 0 & 1 & 1 & 1 & 0 & 0 & 0 & 0 & 0 & 0 & 0 \\
\hline PGS-17 & 0 & 0 & 0 & 1 & 1 & 1 & 0 & 0 & 0 & 0 & 0 & 0 & 0 \\
\hline PGS-18 & 0 & 0 & 0 & 1 & 1 & 1 & 0 & 0 & 0 & 0 & 0 & 0 & 0 \\
\hline PGS-19 & 0 & 0 & 0 & 1 & 1 & 1 & 0 & 0 & 0 & 0 & 0 & 0 & 0 \\
\hline PGS-20 & 0 & 0 & 0 & 1 & 1 & 1 & 0 & 0 & 0 & 0 & 0 & 0 & 0 \\
\hline PGS-21 & 1 & 1 & 1 & 1 & 1 & 1 & 1 & 1 & 1 & 1 & 0 & 0 & 0 \\
\hline PGS-22 & 0 & 1 & 0 & 1 & 1 & 1 & 1 & 0 & 1 & 0 & 0 & 0 & 0 \\
\hline PGS-23 & 0 & 0 & 0 & 1 & 1 & 1 & 1 & 0 & 1 & 0 & 0 & 0 & 0 \\
\hline PGS-24 & 0 & 0 & 0 & 1 & 1 & 1 & 0 & 0 & 0 & 0 & 0 & 0 & 0 \\
\hline PGS-25 & 1 & 1 & 1 & 1 & 1 & 1 & 1 & 1 & 1 & 1 & 0 & 0 & 0 \\
\hline PGS-26 & 1 & 1 & 1 & 1 & 1 & 1 & 1 & 1 & 1 & 1 & 0 & 0 & 0 \\
\hline PGS-27 & 1 & 1 & 1 & 1 & 1 & 1 & 1 & 1 & 1 & 0 & 0 & 0 & 0 \\
\hline PGS-28 & 0 & 0 & 0 & 1 & 1 & 1 & 0 & 0 & 0 & 0 & 0 & 0 & 0 \\
\hline PGS-29 & 0 & 1 & 0 & 1 & 1 & 1 & 1 & 1 & 1 & 1 & 0 & 0 & 0 \\
\hline PGS-30 & 0 & 0 & 0 & 1 & 1 & 1 & 1 & 1 & 1 & 1 & 0 & 0 & 0 \\
\hline PGS-31 & 0 & 1 & 0 & 1 & 1 & 1 & 0 & 0 & 0 & 0 & 0 & 0 & 0 \\
\hline PGS-32 & 0 & 0 & 0 & 1 & 1 & 1 & 0 & 0 & 0 & 0 & 0 & 0 & 0 \\
\hline PGS-33 & 0 & 0 & 0 & 1 & 1 & 1 & 0 & 0 & 0 & 0 & 0 & 0 & 0 \\
\hline PGS-34 & 0 & 0 & 0 & 1 & 1 & 1 & 0 & 0 & 0 & 0 & 0 & 0 & 0 \\
\hline PGS-35 & 0 & 0 & 0 & 1 & 1 & 1 & 0 & 0 & 0 & 0 & 0 & 0 & 0 \\
\hline PGS-36 & 0 & 0 & 0 & 1 & 1 & 1 & 0 & 0 & 0 & 0 & 0 & 0 & 0 \\
\hline PGS-37 & 0 & 0 & 0 & 1 & 1 & 1 & 0 & 0 & 0 & 0 & 0 & 0 & 0 \\
\hline PGS-38 & 0 & 0 & 0 & 1 & 1 & 1 & 0 & 0 & 0 & 0 & 0 & 0 & 0 \\
\hline PGS-39 & 0 & 0 & 0 & 1 & 1 & 1 & 0 & 0 & 0 & 0 & 0 & 0 & 0 \\
\hline PGS-40 & 0 & 0 & 0 & 1 & 1 & 1 & 0 & 0 & 0 & 0 & 0 & 0 & 0 \\
\hline PGS-41 & 0 & 0 & 0 & 1 & 1 & 1 & 0 & 0 & 0 & 0 & 0 & 0 & 0 \\
\hline PGS-42 & 0 & 0 & 0 & 1 & 1 & 1 & 0 & 0 & 0 & 0 & 0 & 0 & 0 \\
\hline PGS-43 & 0 & 0 & 0 & 1 & 1 & 1 & 0 & 0 & 0 & 0 & 0 & 0 & 0 \\
\hline PGS-44 & 0 & 0 & 0 & 1 & 1 & 1 & 0 & 0 & 0 & 0 & 0 & 0 & 0 \\
\hline PGS-45 & 0 & 0 & 0 & 1 & 1 & 1 & 0 & 0 & 0 & 0 & 0 & 0 & 0 \\
\hline PGS-46 & 0 & 0 & 0 & 1 & 1 & 1 & 0 & 0 & 0 & 0 & 0 & 0 & 0 \\
\hline PGS-47 & 0 & 0 & 0 & 1 & 1 & 1 & 0 & 0 & 0 & 0 & 0 & 0 & 0 \\
\hline PGS-48 & 0 & 0 & 0 & 1 & 1 & 1 & 0 & 0 & 0 & 0 & 0 & 0 & 0 \\
\hline PGS-49 & 0 & 0 & 0 & 1 & 1 & 1 & 0 & 0 & 0 & 0 & 0 & 0 & 0 \\
\hline PGS-50 & 0 & 0 & 0 & 1 & 1 & 1 & 0 & 0 & 0 & 0 & 0 & 0 & 0 \\
\hline PGS-51 & 0 & 0 & 0 & 1 & 1 & 1 & 0 & 0 & 0 & 0 & 0 & 0 & 0 \\
\hline PGS-52 & 0 & 0 & 0 & 1 & 1 & 1 & 0 & 0 & 0 & 0 & 0 & 0 & 0 \\
\hline PGS-53 & 1 & 1 & 1 & 1 & 1 & 1 & 1 & 1 & 1 & 0 & 0 & 0 & 0 \\
\hline PGS-54 & 0 & 0 & 0 & 1 & 1 & 1 & 0 & 0 & 0 & 0 & 0 & 0 & 0 \\
\hline PGS-55 & 0 & 0 & 0 & 1 & 1 & 1 & 0 & 0 & 0 & 0 & 0 & 0 & 0 \\
\hline PGS-56 & 0 & 0 & 0 & 1 & 1 & 1 & 0 & 0 & 0 & 0 & 0 & 0 & 0 \\
\hline PGS-57 & 0 & 0 & 0 & 1 & 1 & 1 & 0 & 0 & 0 & 0 & 0 & 0 & 0 \\
\hline
\end{tabular}




\begin{tabular}{|c|c|c|c|c|c|c|c|c|c|c|c|c|c|}
\hline Isolate & AMP & AMX & CHL & CIP & PEF & NAL & SMX & TMP & SXT & TCY & AZM & CFM & CTR \\
\hline PGS-58 & 0 & 0 & 0 & 1 & 1 & 1 & 0 & 0 & 0 & 0 & 0 & 0 & 0 \\
\hline PGS-59 & 0 & 0 & 0 & 1 & 1 & 1 & 0 & 0 & 0 & 0 & 0 & 0 & 0 \\
\hline PGS-60 & 0 & 0 & 0 & 1 & 1 & 1 & 0 & 0 & 0 & 0 & 0 & 0 & 0 \\
\hline PGS-61 & 0 & 0 & 0 & 1 & 1 & 1 & 0 & 0 & 0 & 0 & 0 & 0 & 0 \\
\hline PGS-62 & 0 & 0 & 0 & 1 & 1 & 1 & 0 & 0 & 0 & 0 & 0 & 0 & 0 \\
\hline PGS-63 & 0 & 0 & 0 & 1 & 1 & 1 & 0 & 0 & 0 & 0 & 0 & 0 & 0 \\
\hline PGS-64 & 0 & 0 & 0 & 1 & 1 & 1 & 0 & 0 & 0 & 0 & 0 & 0 & 0 \\
\hline PGS-65 & 0 & 0 & 0 & 1 & 1 & 1 & 0 & 0 & 0 & 0 & 0 & 0 & 0 \\
\hline PGS-66 & 0 & 0 & 0 & 1 & 1 & 1 & 0 & 0 & 0 & 0 & 0 & 0 & 0 \\
\hline PGS-67 & 0 & 0 & 0 & 1 & 1 & 1 & 0 & 0 & 0 & 0 & 0 & 0 & 0 \\
\hline PGS-68 & 0 & 0 & 0 & 1 & 1 & 1 & 0 & 0 & 0 & 0 & 0 & 0 & 0 \\
\hline PGS-69 & 1 & 1 & 1 & 1 & 1 & 1 & 1 & 1 & 1 & 0 & 0 & 0 & 0 \\
\hline PGS-70 & 0 & 0 & 0 & 1 & 1 & 1 & 0 & 0 & 0 & 0 & 0 & 0 & 0 \\
\hline PGS-71 & 0 & 0 & 0 & 1 & 1 & 1 & 0 & 0 & 0 & 0 & 0 & 0 & 0 \\
\hline PGS-72 & 0 & 0 & 0 & 1 & 1 & 1 & 0 & 0 & 0 & 0 & 0 & 0 & 0 \\
\hline PGS-73 & 0 & 0 & 0 & 1 & 1 & 1 & 0 & 0 & 0 & 0 & 0 & 0 & 0 \\
\hline PGS-74 & 0 & 0 & 0 & 1 & 1 & 1 & 0 & 0 & 0 & 0 & 0 & 0 & 0 \\
\hline PGS-75 & 0 & 0 & 0 & 1 & 1 & 1 & 0 & 0 & 0 & 0 & 0 & 0 & 0 \\
\hline PGS-76 & 0 & 0 & 0 & 1 & 1 & 1 & 0 & 0 & 0 & 0 & 0 & 0 & 0 \\
\hline PGS-77 & 0 & 0 & 0 & 1 & 1 & 1 & 0 & 0 & 0 & 0 & 0 & 0 & 0 \\
\hline PGS-78 & 0 & 0 & 0 & 1 & 1 & 1 & 0 & 0 & 0 & 0 & 0 & 0 & 0 \\
\hline PGS-79 & 0 & 0 & 0 & 1 & 1 & 1 & 0 & 0 & 0 & 0 & 0 & 0 & 0 \\
\hline PGS-80 & 0 & 0 & 0 & 1 & 1 & 1 & 0 & 0 & 0 & 0 & 0 & 0 & 0 \\
\hline PGS-81 & 0 & 0 & 0 & 1 & 1 & 1 & 0 & 0 & 0 & 0 & 0 & 0 & 0 \\
\hline PGS-82 & 0 & 0 & 0 & 1 & 1 & 1 & 0 & 0 & 0 & 0 & 0 & 0 & 0 \\
\hline PGS-83 & 0 & 0 & 0 & 1 & 1 & 1 & 0 & 0 & 0 & 0 & 0 & 0 & 0 \\
\hline PGS-84 & 0 & 0 & 1 & 1 & 1 & 1 & 0 & 0 & 0 & 0 & 0 & 0 & 0 \\
\hline PGS-85 & 1 & 1 & 1 & 1 & 1 & 1 & 1 & 1 & 1 & 0 & 0 & 0 & 0 \\
\hline PGS-86 & 0 & 0 & 0 & 1 & 1 & 1 & 0 & 0 & 0 & 0 & 0 & 0 & 0 \\
\hline PGS-87 & 0 & 0 & 0 & 1 & 1 & 1 & 0 & 0 & 0 & 0 & 0 & 0 & 0 \\
\hline PGS-88 & 0 & 0 & 0 & 1 & 1 & 1 & 0 & 0 & 0 & 0 & 0 & 0 & 0 \\
\hline PGS-89 & 0 & 0 & 0 & 1 & 1 & 1 & 0 & 0 & 0 & 0 & 0 & 0 & 0 \\
\hline PGS-90 & 0 & 0 & 0 & 1 & 1 & 1 & 0 & 0 & 0 & 0 & 0 & 0 & 0 \\
\hline PGS-91 & 0 & 0 & 0 & 1 & 1 & 1 & 0 & 0 & 0 & 0 & 0 & 0 & 0 \\
\hline PGS-92 & 0 & 0 & 0 & 1 & 1 & 1 & 0 & 0 & 0 & 0 & 0 & 0 & 0 \\
\hline PGS-93 & 0 & 0 & 0 & 1 & 1 & 1 & 0 & 0 & 0 & 0 & 0 & 0 & 0 \\
\hline PGS-94 & 0 & 0 & 0 & 1 & 1 & 1 & 0 & 0 & 0 & 0 & 0 & 0 & 0 \\
\hline PGS-95 & 0 & 0 & 0 & 1 & 1 & 1 & 0 & 0 & 0 & 0 & 0 & 0 & 0 \\
\hline PGS-96 & 0 & 0 & 0 & 1 & 1 & 1 & 0 & 0 & 0 & 0 & 0 & 0 & 0 \\
\hline PGS-97 & 0 & 0 & 0 & 1 & 1 & 1 & 0 & 0 & 0 & 0 & 1 & 0 & 0 \\
\hline PGS-98 & 0 & 0 & 1 & 1 & 1 & 1 & 0 & 0 & 0 & 0 & 0 & 0 & 0 \\
\hline PGS-99 & 1 & 1 & 1 & 1 & 1 & 1 & 1 & 1 & 1 & 0 & 0 & 0 & 0 \\
\hline PGS-100 & 0 & 0 & 0 & 1 & 1 & 1 & 0 & 0 & 0 & 0 & 0 & 0 & 0 \\
\hline PGS-101 & 0 & 0 & 0 & 1 & 1 & 1 & 0 & 0 & 0 & 0 & 0 & 0 & 0 \\
\hline PGS-102 & 1 & 1 & 1 & 1 & 1 & 1 & 1 & 1 & 1 & 0 & 0 & 0 & 0 \\
\hline PGS-103 & 0 & 0 & 0 & 1 & 1 & 1 & 0 & 0 & 0 & 0 & 0 & 0 & 0 \\
\hline PGS-104 & 0 & 0 & 0 & 1 & 1 & 1 & 0 & 0 & 0 & 0 & 0 & 0 & 0 \\
\hline PGS-105 & 0 & 0 & 0 & 1 & 1 & 1 & 0 & 0 & 0 & 0 & 0 & 0 & 0 \\
\hline PGS-106 & 0 & 0 & 0 & 1 & 1 & 1 & 0 & 0 & 0 & 0 & 0 & 0 & 0 \\
\hline PGS-107 & 0 & 0 & 0 & 1 & 1 & 1 & 0 & 0 & 0 & 0 & 0 & 0 & 0 \\
\hline PGS-108 & 0 & 0 & 0 & 1 & 1 & 1 & 0 & 0 & 0 & 0 & 0 & 0 & 0 \\
\hline PGS-109 & 0 & 0 & 0 & 1 & 1 & 1 & 0 & 0 & 0 & 0 & 0 & 0 & 0 \\
\hline PGS-110 & 0 & 0 & 0 & 1 & 1 & 1 & 0 & 0 & 0 & 0 & 0 & 0 & 0 \\
\hline PGS-111 & 0 & 0 & 0 & 1 & 1 & 1 & 0 & 0 & 0 & 0 & 0 & 0 & 0 \\
\hline PGS-112 & 0 & 0 & 0 & 1 & 1 & 1 & 0 & 0 & 0 & 0 & 0 & 0 & 0 \\
\hline PGS-113 & 0 & 0 & 0 & 1 & 1 & 1 & 0 & 0 & 0 & 0 & 0 & 0 & 0 \\
\hline PGS-114 & 0 & 0 & 0 & 1 & 1 & 1 & 0 & 0 & 0 & 0 & 0 & 0 & 0 \\
\hline
\end{tabular}




\begin{tabular}{|l|l|l|l|l|l|l|l|l|l|l|l|l|l|}
\hline Isolate & AMP & AMX & CHL & CIP & PEF & NAL & SMX & TMP & SXT & TCY & AZM & CFM & CTR \\
\hline PGS-115 & 0 & 0 & 0 & 1 & 1 & 1 & 0 & 0 & 0 & 0 & 0 & 0 & 0 \\
\hline PGS-116 & 0 & 0 & 0 & 1 & 1 & 1 & 0 & 0 & 0 & 0 & 0 & 0 & 0 \\
\hline PGS-117 & 1 & 1 & 1 & 1 & 1 & 1 & 1 & 1 & 1 & 0 & 0 & 0 & 0 \\
\hline PGS-118 & 1 & 1 & 1 & 1 & 1 & 1 & 1 & 1 & 1 & 0 & 0 & 0 & 0 \\
\hline PGS-119 & 0 & 0 & 0 & 1 & 1 & 1 & 0 & 0 & 0 & 0 & 0 & 0 & 0 \\
\hline PGS-120 & 0 & 0 & 0 & 1 & 1 & 1 & 0 & 0 & 0 & 0 & 0 & 0 & 0 \\
\hline PGS-121 & 0 & 0 & 0 & 1 & 1 & 1 & 0 & 0 & 0 & 0 & 0 & 0 & 0 \\
\hline PGS-122 & 0 & 0 & 0 & 1 & 1 & 1 & 0 & 0 & 0 & 0 & 0 & 0 & 0 \\
\hline PGS-123 & 0 & 0 & 0 & 1 & 1 & 0 & 0 & 0 & 0 & 0 & 0 & 0 & 0 \\
\hline PGS-124 & 0 & 0 & 0 & 1 & 1 & 1 & 0 & 0 & 0 & 0 & 0 & 0 & 0 \\
\hline PGS-125 & 0 & 0 & 0 & 1 & 1 & 1 & 0 & 0 & 0 & 0 & 0 & 0 & 0 \\
\hline PGS-126 & 0 & 0 & 0 & 1 & 1 & 1 & 0 & 0 & 0 & 0 & 0 & 0 & 0 \\
\hline PGS-127 & 1 & 1 & 1 & 1 & 1 & 1 & 1 & 1 & 1 & 1 & 0 & 0 & 0 \\
\hline PGS-128 & 0 & 0 & 0 & 1 & 1 & 1 & 0 & 0 & 0 & 0 & 0 & 0 & 0 \\
\hline PGS-129 & 0 & 0 & 0 & 1 & 1 & 1 & 0 & 0 & 0 & 0 & 0 & 0 & 0 \\
\hline PGS-130 & 0 & 0 & 0 & 1 & 1 & 1 & 0 & 0 & 0 & 0 & 0 & 0 & 0 \\
\hline PGS-131 & 0 & 0 & 0 & 1 & 1 & 1 & 1 & 1 & 1 & 1 & 0 & 0 & 0 \\
\hline PGS-132 & 0 & 0 & 0 & 1 & 1 & 1 & 0 & 0 & 0 & 0 & 0 & 0 & 0 \\
\hline PGS-133 & 1 & 1 & 1 & 1 & 1 & 1 & 1 & 1 & 1 & 1 & 0 & 0 & 0 \\
\hline
\end{tabular}

Table 1. Antimicrobial resistance profile of 133 Salmonella isolates. *Antibiotic abbreviations are as follows: AMP, Ampicillin; AMX, Amoxicillin; CHL, Chloramphenicol; CIP, Ciprofloxacin; PEF, Peflox; NAL,

Nalidixic Acid; SMX, Sulfamethoxazole; TMP, Trimethoprim; SXT, Cotrimoxazole; TCY, Tetracycline; AZM, Azithromycin; CFM, Cefixime; CTR, Ceftriaxone; Zero, Susceptible; One, Resistance.

\begin{tabular}{|l|l|l|l|}
\hline $\begin{array}{l}\text { AMR } \\
\text { gene }\end{array}$ & AMR SNP's & Resistance type & Frequency \\
\hline gyrA & $\begin{array}{l}\text { D87N, S83Y, D87Y, } \\
\text { S83F, D87G }\end{array}$ & fluoroquinolone & $94.74 \%$ \\
\hline gyrB & S464F, A574V & fluoroquinolone & $6.77 \%$ \\
\hline parC & E84G, E84K, S80I & fluoroquinolone & $21.05 \%$ \\
\hline parE & $\begin{array}{l}\text { A364V, D420N, } \\
\text { L416F }\end{array}$ & fluoroquinolone & $11.28 \%$ \\
\hline acrB & R717Q & azithromycin & $0.75 \%$ \\
\hline
\end{tabular}

Table 2. Chromosomal point mutations associated with the quinolone resistance-determining region (QRDR) and azithromycin resistance for $S$. Typhi.

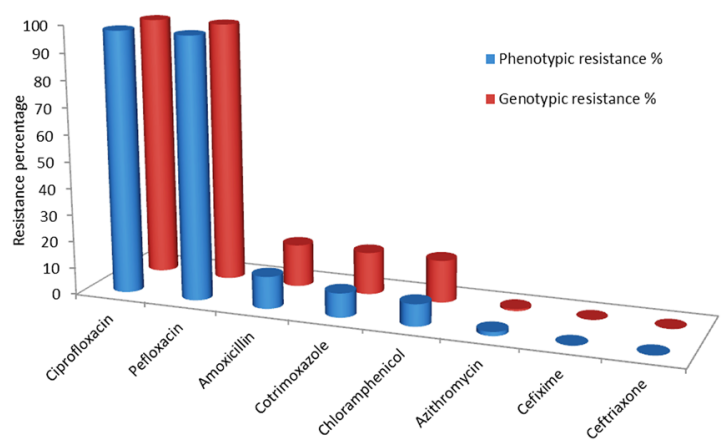

Figure 4. Genotypes-Phenotypes Correlation. Schematic representation of correlation between genotype and phenotype obtained from WGS and disk diffusion method, respectively. Overall correlation of genotypic and phenotypic was $91.83 \%$ and $99.06 \%$ for sensitivity and specificity, respectively. 


\begin{tabular}{|l|l|l|l|l|l|l|}
\hline & \multicolumn{2}{|l|}{ Phenotypically Non-susceptible } & \multicolumn{2}{l|}{ Phenotypically Susceptible } & Sensitivity \\
\cline { 2 - 6 } Antibiotic & $\begin{array}{l}\text { Genotypically } \\
\text { Non-susceptible }\end{array}$ & $\begin{array}{l}\text { Genotypically } \\
\text { Susceptible }\end{array}$ & $\begin{array}{l}\text { Genotypically } \\
\text { Non-susceptible }\end{array}$ & $\begin{array}{l}\text { Genotypically } \\
\text { susceptible }\end{array}$ \\
\hline Ciprofloxacin & 129 & 1 & 0 & 3 & 99.23 & 100 \\
\hline Pefloxacin & 129 & 1 & 0 & 3 & 99.23 & 100 \\
\hline Amoxicillin & 16 & 0 & 5 & 112 & 100 & 95.73 \\
\hline Chloramphenicol & 11 & 0 & 10 & 112 & 100 & 91.80 \\
\hline Co-trimoxazole & 12 & 0 & 9 & 112 & 100 & 92.56 \\
\hline Cefixime & 0 & 0 & 0 & 133 & 100 & 100 \\
\hline Ceftriaxone & 0 & 0 & 0 & 133 & 100 & 100 \\
\hline Azithromycin & 1 & 1 & 0 & 131 & 50 & 100 \\
\hline Overall & & & & & $\mathbf{9 3 . 5 6}$ & $\mathbf{9 7 . 5 1}$ \\
\hline
\end{tabular}

Table 3. Genotypes-phenotypes correlation prediction for all 133 Salmonella isolates in the study.

Fluoroquinolones resistance. Molecular determinants of resistance to fluoroquinolone including ciprofloxacin and pefloxacin antibiotics encoded by $g y r \mathrm{~A}, \operatorname{gyrB}$, parC, and parE genes were detected in $96.99 \%$ strains $(129 / 133)$ by WGS. Mutations in $g y r A$, parC, parE and gyrB was observed in $94.74 \%, 21.05 \%, 5.26 \%$ and $2.26 \%$ of strains, respectively (Tables 1-2; Table S5-S6 in Supplementary File 1). The identified genes were associated with mutations in Quinolone Resistance Determining Region of DNA gyrase enzyme, the binding site for fluoroquinolone. Antimicrobial resistance to fluoroquinolones was $97.74 \%(130 / 133)$ by both disc diffusion and E-test method. MIC distribution ranged between 2-24 mg/L and peaked at $12 \mathrm{mg} / \mathrm{L}$. A gradual increase in decreased susceptibility to ciprofloxacin was demonstrated in a longitudinal analysis of past 20 years ${ }^{44}$. DNA Gyrase A mutations at position 83 (Ser-83 $\rightarrow$ Phe and Ser-83 $\rightarrow$ Tyr) are the most prevalent resistance mechanisms for fluoroquinolone in India ${ }^{45}$, followed by Ser- $80 \rightarrow$ Ile substitution in $\operatorname{par} C$ gene. We also found one fluoroquinolone susceptible strains with mutation in gyrA gene which might be occurring due to bacterial promoter modifications leading to the overexpression of other DNA topoisomerases. Highly non-susceptible strains (with ciprofloxacin MIC $>8 \mathrm{mg} / \mathrm{L}$ ) were found to be double or triple mutants with mutations in gyrA83, gyrA87 and parC80. Strains with moderate resistance to ciprofloxacin possessed single mutations in DNA gyrA gene at Ser83 position. The observed sensitivity was $99.23 \%$ and specificity was $100 \%$ for both ciprofloxacin and peflox resistance (Fig. 4; Table 3; Table S7 in Supplementary File 1).

\section{Cephalosporins resistance.}

- Antimicrobial susceptibility to antibiotics, cefixime and ceftriaxone, observed for all strains is consistent with other studies from India ${ }^{1,32}$. Resistance determinants were also not found for cephalosporins (Table 1; Table S5 in Supplementary File 1). Though all the strains were susceptible, however, a gradual increase in median MIC values was perceived over a time period. This clearly raises an alarm towards the judicial use of these antibiotics.

Azithromycin resistance. Only $0.75 \%$ non-susceptible strains (1/133) to azithromycin were observed by WGS as compared to $1.50 \%$ (2/133) strains by phenotypic method. The non-susceptible strains harboured mutations in the $a c r B$ efflux pump regulator genes that have been known to confer macrolide resistance ${ }^{46}$. In this study, we detected R717Q mutation on AcrB efflux pump as a cause of azithromycin resistance in $S$. Typhi ${ }^{11}$ (Tables 1-2; Table S5-S6 in Supplementary File 1). AcrB-R717Q precisely leads to increased macrolide resistance. This study indicated that most of the $S$. Typhi strains were susceptible to azithromycin. These strains were screened separately for presence of macrolide resistance determinants ( $m p h A$, ermA, ere $A$, ere $B, m e f A$ and $m s r A$ genes) by PCR and presence of mutations in $r l p D$ and $r l p V$, where also no macrolide resistance mechanism was observed $^{46}$. The sensitivity was $50 \%$ and specificity was $100 \%$ for azithromycin resistance (Fig. 4; Table 3; Table S7 in Supplementary File 1).

Genotypic resistance to other antimicrobial agents. S. Typhi can demonstrate resistance to multiple antibiotics by acquiring new resistance genes through horizontal genes transfer (HGT). The acquired antimicrobial resistance genes including $a a c\left(6^{\prime}\right)-I a a, A A C\left(6^{\prime}\right)-I y$, aadA1, aph (3")-Ib, aph(6)-Id, strA, and strB that provided resistance to aminoglycosides were observed in $100 \%$ (133/133) isolates (Table 1; Table S5 in Supplementary File 1). Tetracycline resistance encoded by $\operatorname{tet}(A)$, tet $(B)$, and $\operatorname{tet}(R)$ genes for tetracycline efflux pumps were detected mainly in $9.02 \%$ strains (12/133) of $S$. Typhi (Table 1; Table S5 in Supplementary File 1). In addition, $S$. Typhi isolates harboured the genes baeR, emrb, $H$-NS, marA, $m d f A, m d t K, m s b A$, acrA, emrR, kpnE, kpnF, marR, sdiA, crp, soxR, and soxS that could confer multidrug resistance and were detected in all 133 strains (Table S5 in Supplementary File 1). The mdsABC complex, a multidrug transporter of Salmonella, comprising $m d s A, m d s B$, and $m d s C$ units was observed in every single isolate. The mdsABC complex is recognized to contribute resistance against a diverse set of drugs and toxins ${ }^{47}$. The identified multi-efflux pump $m d t K$ gene, conferring resistance against the drugs, acriflavin, doxorubicin and norfloxacin, was observed in $100 \%(133 / 133)$ of the isolates ${ }^{48}$. The gene, sdiA, a multi-drug resistance pump regulator for AcraB, was also present in 100\% (133/133) of the isolates ${ }^{49}$. The pathogenicity and resistance profile of the various Salmonella isolates can be attributed to the presence of identified genes. 


\begin{tabular}{|l|l|l|l|l|l|l|l|l|l|l|l|l|l|l|}
\hline Genotypes & $\begin{array}{l}\text { No. of } \\
\text { isolates } \\
(\%)\end{array}$ & $\begin{array}{l}\text { MLST } \\
\text { ST }\end{array}$ & $\begin{array}{l}\text { gyrA } \\
\text { D87N }\end{array}$ & $\begin{array}{l}\text { gyrA } \\
\text { S83Y }\end{array}$ & $\begin{array}{l}\text { gyrA } \\
\text { D87Y }\end{array}$ & $\begin{array}{l}\text { gyrA } \\
\text { S83F }\end{array}$ & $\begin{array}{l}\text { gyrA } \\
\text { D87G }\end{array}$ & $\begin{array}{l}\text { gyrB } \\
\text { S464F }\end{array}$ & $\begin{array}{l}\text { parC } \\
\text { S80I }\end{array}$ & $\begin{array}{l}\text { parC } \\
\text { E84G }\end{array}$ & $\begin{array}{l}\text { parC } \\
\text { E84K }\end{array}$ & $\begin{array}{l}\text { parE } \\
\text { D420N }\end{array}$ & $\begin{array}{l}\text { parE } \\
\text { L416F }\end{array}$ & Inc Types \\
\hline 2.2 & $4(3.01)$ & 2 & 0 & 0 & 0 & 1 & 0 & 0 & 0 & 0 & 0 & 0 & 0 & \\
\hline 2.5 & $3(2.26)$ & 2 & 0 & 0 & 0 & 1 & 1 & 0 & 0 & 0 & 0 & 0 & 0 & \\
\hline 3 & $1(0.75)$ & 1 & 0 & 0 & 0 & 1 & 0 & 0 & 0 & 0 & 0 & 0 & 0 & \\
\hline 3.3 & $4(3.01)$ & 2 & 0 & 0 & 0 & 1 & 0 & 0 & 0 & 0 & 0 & 0 & 0 & IncFIB(pHCM2) \\
\hline 2.2 .2 & $2(1.50)$ & 2 & 0 & 1 & 0 & 0 & 0 & 0 & 0 & 0 & 0 & 0 & 1 & \\
\hline 2.2 .4 & $1(0.75)$ & 2 & 0 & 1 & 0 & 0 & 0 & 0 & 0 & 0 & 0 & 0 & 0 & \\
\hline 3.3 .1 & $6(4.51)$ & 2 & 0 & 1 & 0 & 0 & 0 & 0 & 0 & 0 & 0 & 0 & 1 & \\
\hline 3.3 .2 & $3(2.26)$ & 2 & 0 & 1 & 1 & 0 & 0 & 0 & 0 & 0 & 0 & 0 & 0 & \\
\hline 4.3 .1 & $19(14.29)$ & 1 & 0 & 1 & 0 & 1 & 1 & 1 & 0 & 0 & 0 & 1 & 0 & IncFIA(HI1); IncHI1A/IncHI1B(R27) \\
\hline 4.3 .1 .1 & $18(13.53)$ & 1 & 0 & 1 & 0 & 1 & 1 & 1 & 0 & 0 & 1 & 0 & 0 & $\begin{array}{l}\text { IncHI1A/IncHI1B(R27); } \\
\text { IncFIB(pHCM2); Col(BS512) }\end{array}$ \\
\hline 4.3 .1 .2 & $72(54.14)$ & 1 & 1 & 1 & 0 & 1 & 0 & 0 & 1 & 1 & 1 & 0 & 0 & $\begin{array}{l}\text { IncFIB(pHCM2); IncN; p0111; } \\
\text { IncHI1A/IncHI1B(R27) }\end{array}$ \\
\hline
\end{tabular}

Table 4. Genotype-associated sequence types (ST), mutation patterns in the genes encoding DNA gyrase ( $g y r A$ and $g y r B$ ) or topoisomerase (parC and parE) and known plasmid Inc types in S. Typhi.

MLST and cgMLST-based phylogenetic analysis. Whole genome sequencing-based in silico multi-locus sequence typing (MLST) profile disclosed low genetic variation in housekeeping genes (aroC, dnaN, hemD, hisD, purE, sucA, and thrA) among 133 Salmonella isolates. Two different sequence types (STs) including ST1 and ST2, irrespective the year of isolation were observed ${ }^{49}$. ST1 was the predominant type, accounting for $81.95 \%$ (109/133) of examined strains, whereas ST2 was observed in $17.29 \%$ (23/133) of the strains (Table 4; Table S8 in Supplementary File 1). In addition, one novel ST, aroC1 was identified which consists of a missense substitution $(\mathrm{C}->\mathrm{T})$ compared to the predominant aro $C 1$ allele present in almost all $S$. Typhi strains. MLST analysis revealed the presence of ST1 in all strains, irrespective of the geographical location, whereas ST2 was completely absent in strains isolated at Chennai and Wardha city of India. We next investigated the epidemiological outbreak using the phylogenetic tree based on gene-by-gene-based core genome MLST (cgMLST) method. The analysis yielded a total of 3002 core loci, where the number of successfully called alleles per isolate ranged from $2664(88.74 \%)$ to 2706 (90.14\%) depending on the gene. For high-resolution subtyping, core-genome sequence types (cgST) were considered, where the allele matches in cgST per isolate ranged from $2634(87.74 \%)$ to $2699(89.91 \%)$. The cgST-based tree showed that isolates with identical ST1 (119 strains) and novel ST (1 strain) were clustered in one group, whereas isolates with ST2 (23 strains) were separated into outlier cluster (Fig. S2 in Supplementary File 3; Table S9 in Supplementary File 1). Inter-strain diversity among all isolates yielded the Simpson's diversity index (SDI) equal to 0.51 . The result demonstrates a lower level of genetic diversity and epidemiological links among isolates of $S$. Typhi.

Population structure and QRDR mutation patterns. The genotyphi classification according to the scheme of Wong et al..$^{50}$, revealed 11 distinct genotypes $(2.2,2.2 .2,2.2 .4,2.5,3,3.3,3.3 .1,3.3 .2,4.3 .1,4.3 .1 .1$, and 4.3.1.2) signifying the diverse population structure in India (Table 4; Table S8 in Supplementary File 1). The majority (81.95\%) of the isolates belonged to H58 clade including H58 lineage (genotype 4.3.1), H58 lineage I (genotype 4.3.1.1) and H58 lineage II (genotype 4.3.1.2) ${ }^{23,51-54}$. The H58 lineage (genotype 4.3.1) was present in $14.29 \%$ (19/133) of Salmonella isolates obtained in India from January 1993 to December 2016 and probably emerged from South Asia and East Africa in the initial 1990s $\mathrm{s}^{54,55}$. H58 lineage I (genotype 4.3.1.1) was present in $13.53 \%$ (18/133) of the isolates, whereas H58 lineage II (genotype 4.3.1.2) was dominant in the majority of the isolates (70/133, 52.63\%). The H58 lineage II isolates appeared to be dominant in Nepal, India and Pakistan ${ }^{12,51,52}$, whereas it was rare in nearby country Bangladesh ${ }^{56}$. In contrast, H58 lineage I (genotype 4.3.1.1) isolates were dominant (31.2\%) in Bangladesh compared to India (13.53\%). The genotypes 3, 3.3, 3.3.1 and 3.3.2 were collectively observed for $10.53 \%$ of Salmonella isolates, whereas genotypes 2.2, 2.2.2 and 2.2.4 were found in 7.52\% of isolates. The reference-based (Fig. S3 in Supplementary File 4) and recombination free (Fig. S4 in Supplementary File 5) phylogenetic analysis was carried out separately to locate outbreak clusters based on SNPs shared among $S$. Typhi isolates collected from diverse geographical locations $s^{55}$. The tree showed that the strains were mainly grouped into two clusters, where $17.29 \%$ of strains (23/133) clustered close together with a distinct segregation from others strains (109/133). The phylogenies revealed that $93.39 \%$ of the reference genome (S. Typhi CT18) was covered in all isolates. The minimum and maximum SNPs differences in comparison to reference genome was observed for PGS-123 (275 SNPs) and PGS-66 (521 SNPs), respectively (Table S10 in Supplementary File 1). SNP tree was further explored to investigate the distribution and mutation patterns in the genes encoding DNA gyrase ( $g y r A$ and $g y r B)$ or topoisomerase ( $\operatorname{parC}$ and $p a r E$ ) that reside in the quinolone resistance-determining region (QRDR). The overall high genotypic (96.99\%) and phenotypic $(97.74 \%)$ resistance to ciprofloxacin and pefloxacin was observed owing to QRDR mutants in Salmonella isolates (Fig. 4; Table S6 in Supplementary File 1). This is similar to the previously reported cases where the QRDR mutants were dominant in India (97\%), Bangladesh (94\%), Nepal (66\%), Pakistan (94\%), Myanmar (100\%), Uganda (100\%), and Nigeria (50\%) $)^{54}$. The close examination of the tree exposed the genotype-based clustering in $S$. Typhi. The minimum and maximum numbers of SNP differences within the cluster strains were observed to be low as compared to strains belonging to different 
clusters and reference strain. The isolates belonging to genotype 3.3.2 were clustered in two clades, each with a different mutation (S83Y; D87Y) in the Quinolone Resistance Determining Region (QRDR) of gene gyrA as conveyed in Bangladesh ${ }^{56}$. QRDR double mutation in gyrA (S83F) and parE (L416F) was commonly observed in the cluster of genotype 3.3.1 which is linked with travel to West Africa ${ }^{54}$. QRDR triple mutants, resistant to ciprofloxacin was observed in genotype 4.3.1 (gyrA_S83Y, S83F, D87G; gyrB_S464F; parE_D420N) and genotype 4.3.1.1 (gyrA_S83Y, S83F, D87G; gyrB_S464F; parC_E84K) which were known to be associated with travel to India $^{54}$. Triple mutant ( $g y r A-S 83 F, g y r A-D 87 N$ and parC-S80I) conferred resistance to ciprofloxacin in $S$. Typhi were common in the cases reported with travel to India, Pakistan and Nepal. The mutation in gyrA (S83F) was commonly observed in seven genotypes $2.2,2.5,3,3.3,4.3 .1,4.3 .1 .1$, and 4.3.1.2. Similarly, shared mutation in gyrA (S83Y) was observed between seven genotypes namely 2.2.2, 2.2.4, 3.3.1, 3.3.2, 4.3.1, 4.3.1.1, and 4.3.1.2. The mutation frequency of $g y r A \_S 83 \mathrm{~F}(\mathrm{n}=74 ; 55.64 \%)$ observed in our data was accordance with previously reported cases of travel to South Asia including India $(n=71)$, Pakistan $(n=134)$, and Bangladesh $(n=25)$, and in contrast with travel history to Central Asia $(n=1)$, Europe $(n=1)$, Middle East $(n=1)$, and South America $(\mathrm{n}=1)^{54}$. The mutation frequency of $g y r A$ S83Y $(\mathrm{n}=48 ; 36.09 \%)$ was similar with previously discussed cases of travel to India $(n=56)$. The shared QRDR mutations between $S$. Typhi lineages in the phylogeny indicated that they might have inherited from a shared common ancestor. Genotype-associated mutation patterns are given in Table 4 and Fig. S4 in Supplementary File 5.

\section{Methods}

Study design. This study was conducted on clinical strains isolated from the blood culture of patients presented with typhoid fever from January 1993 to December 2016. We used blood culture isolates from bacteriology laboratory, A.I.I.M.S., New Delhi, where the samples were sent as a part of routine diagnosis. All the procedures were performed as per CLSI guidelines. All the experiments and data analysis were completed at All India Institute of Medical Science (A.I.I.M.S.), New Delhi.

Bacterial isolates. Clinical isolates of $S$. Typhi were revived from the archived cryopreserved collection of blood culture isolates and identified by standard biochemical and serological methods as described earlier ${ }^{57}$. The collection comprised 686 strains from which 133 strains of S. Typhi were selected using random number generator function in Stata v.14.1 (StataCorp, College Station, TX, USA) ${ }^{58}$. The strains were serially named as PGS1 (Pan Genome Study) to PGS133 for simplicity in data analysis.

Whole genome sequencing. Genomic DNA isolated from freshly grown overnight culture (QIAamp DNA minikit; Qiagen, Germany) was quantified using Qubit fluorometer (Life Technologies, USA). Sequencing library preparation was by Illumina Nextera XT DNA sample preparation kit (Illumina, USA). Genomic DNA samples were fragmented using Covaris M-series (M220) at temperature of 5.5 to $6^{\circ} \mathrm{C}$ for 40 seconds. DNA fragments were end repaired using dA bases before ligation with Illumina indexed adapters, amplified for 10 cycles of PCR and sequenced employing v2 and v3 chemistry with paired-end $2 \times 151 \mathrm{bp}$ reads on Illumina MiSeq (Illumina, USA). Output data files were de-multiplexed and transformed with Casava v.1.8.2. into FASTQ files (Illumina, Inc, USA).

Preprocessing, genome assembly and analysis. The selected sequence paired-end reads from FastQC v0.11.4 ${ }^{59}$ were pre-processed and assembled de novo with A5-miseq pipeline ${ }^{60}$. Sequence adapters and low-quality $(<\mathrm{Q} 30)$ regions were filtered with trimmomatic v0.36 ${ }^{61}$. Read errors were corrected by SGA's k-mer-based error correction algorithm ${ }^{62}$. Paired and unpaired reads were assembled utilizing IDBA-UD algorithm and quality of genome assembly evaluated by QUAST (http://quast.sourceforge.net/quast) ${ }^{63}$. The redundant homologues with identity cut-off of 0.9 were removed from assembled de novo contigs by CD-HIT ${ }^{64}$. The assembled genomes were aligned to a reference genome of $S$. Typhi to avoid the risk of cross-contamination. The assembled bacterial genomes were annotated with Prokka v1.1265 (http://www.vicbioinformatics.com/software.prokka.shtml). The total numbers of coding sequence regions (CDS) in annotated genomes were compared among all strains to filter out outlier strains.

Pan-genome construction. To identify strain-specific genomic features and genomic diversity among $S$. Typhi isolates, the pan-genome was constructed using computational pipeline BPGA ${ }^{66}$. The entire set of proteins served as input for the BPGA analysis. The clustering of genes into families was generated with USEARCH ${ }^{67}$ with $90 \%$ sequence identity as a cutoff. To reduce the impact of outliers, the core-and pan-genome size was determined for two isolates at first, and then the remaining isolates were added iteratively till the exact contaminant strain liable for shrinking the core-genome size was detected. The outliers were excluded in the final version of pan-genome. In order to evade any bias during the sequential inclusion of genomes, random sequence/order of genomes permutations were performed. The pan-genome functional analysis module of BPGA was used to assign Cluster of Orthologous Genes (COG) ${ }^{35}$ and Kyoto Encyclopedia of Genes and Genomes (KEGG) ${ }^{36}$ classes to the core, accessory, and unique gene families. The results were plotted with Gnuplot and PanGP ${ }^{68,69}$.

Identification of resistance determinants. The resistance genes in the assembled Salmonella genomes were predicted through the resistance gene identifier (RGI) from the Comprehensive Antibiotic Resistance Database (CARD, available at https://card.mcmaster.ca/analyze/rgi) ${ }^{70}$ and Pathogenwatch from the Center for Genomic Pathogen Surveillance (CGPS, available at https://pathogen.watch) databases of antimicrobial resistance genes ${ }^{71}$. We used cut-off criteria of $\geq 50 \%$ sequence identity and $\geq 70 \%$ query coverage. RGI (RGI 4.2.0, CARD 2.0.3) prediction of resistome was determined based on homology and SNP models, where the "perfect and strict hits only" criteria were chosen for the prediction. ResFinder webserver 3.0 (https://cge.cbs.dtu.dk/ 
services/ResFinder/) was used to pinpoint the acquired antimicrobial resistance genes and genes associated with chromosomal point mutations ${ }^{72}$.

Phenotypic antimicrobial susceptibility determination. The testing for antimicrobial susceptibility was through Kirby-Bauer disc diffusion assay for chloramphenicol (CHL; 30 $\mu \mathrm{g}$ ), ampicillin (AMP $10 \mu \mathrm{g}$ ), trimethoprim-sulfamethoxazole (TMP/SMX; 1.25/23.75 $\mu \mathrm{g}$ ) ciprofloxacin (CIP; $5 \mu \mathrm{g}$ ), pefloxacin (PFX; $5 \mu \mathrm{g}$ ), cefixime (CFM; $5 \mu \mathrm{g})$, ceftriaxone (CTR; $30 \mu \mathrm{g})$, and azithromycin (AZM; $15 \mu \mathrm{g}$ ) as per CLSI guidelines ${ }^{73}$. Ampicillin disk diffusion test was used to predict the antimicrobial susceptibility results for amoxicillin as recommended by current CLSI guidelines ${ }^{73}$. Minimum inhibitory concentration (MIC) was determined for ciprofloxacin, ceftriaxone and azithromycin by E-test method (E. test, BioMerieux, France). Quality control for antimicrobial susceptibility was done using Escherichia coli ATCC 25922.

Correlation of susceptibility genotypes and phenotypes. Overall 1064 phenotypic data analysis points detected from 133 strains through phenotypic antimicrobial susceptibility testing were correlated with the existence of corresponding plasmid mediated resistance gene(s) as well as structural gene mutations. Phenotypically intermediate and resistant strains were together referred as non-susceptible in this investigation. Considering phenotypic results as the reference outcome, sensitivity of genomic method of antimicrobial susceptibility determination was calculated by dividing the number of genotypically non-susceptible isolates by the overall sum of isolates displaying phenotypic resistance. Specificity was determined by dividing the number of genotypically susceptible isolates by total number of phenotypically susceptible isolates.

Phylogenetic tree based on cgMLST and SNPs. Whole-genome sequencing (WGS)-based in silico multi-locus sequence typing (MLST) of Salmonella strains were performed using MLST 2.0 server $^{74}$. The gene-by-gene-based core genome MLST (cgMLST) was completed using cgMLSTFinder (version 1.1) server ${ }^{75}$, whereas referenced-based SNP tree using maximum likelihood method was constructed by CSI phylogeny (version 1.4) server ${ }^{76}$ from the Centre for Genomic Epidemiology (www.genomicepidemiology.org). The CSI phylogeny build phylogenetic tree is based on the concatenated alignment of the high-quality SNPs, where SNPs were filtered out if the depth at the SNP position was not at least 10x or at least $10 \%$ of the average depth for the particular genome mapping. SNPs were also filtered out if the mapping quality was below 25 or the SNP quality was below 30. The genotypic profile and recombination free SNP tree was built using Pathogenwatch from the Center for Genomic Pathogen Surveillance (CGPS, available at https://pathogen.watch). The phylogenetic tree was visualized using FigTree v1.4.3 (http://tree.bio.ed.ac.uk/software/figtree/) ${ }^{77}$ and iTOL (https://itol.embl.de/) ${ }^{78}$.

Ethics declarations. The protocol for this study was approved (IEC/NP-283-2012) by the Ethics Committee, All India Institute of Medical Science, New Delhi, India.

\section{Conclusions}

Salmonella Typhi continues to pose a major challenge in treatment as it causes invasive infections and acquires antimicrobial resistance (AMR) genes to become non-susceptible to available drugs ${ }^{79}$. As the pipeline for new antityphoidal drugs are exhausted, it has become imperative to explore whole genomes to understand the characteristics and search for novel diagnostic targets. Findings from this study revealed a high correlation between the phenotypes and their corresponding genotypes. The pan-genome analysis revealed the characteristics of gene pool shared by clinical Salmonella Typhi isolates, indicating that the core genes were enriched in metabolism related function whereas accessory genes were majorly implicated in pathogenesis and antimicrobial resistance mechanisms. The cgMLST and SNP-based phylogenetic analysis revealed lower level of genetic diversity where a larger of the strains belonged to the same outbreak and few were separated into outlier cluster. This work overall highlights the importance of WGS-based resistance gene screening for the tracking of AMR mechanisms in $S$. Typhi and thus can ${ }^{66}$ contribute to the battle against the expanding AMR threat worldwide.

\section{Data availability}

WGS data of all 133 Salmonella isolates have been submitted to the National Center for Biotechnology Information (NCBI; https://submit.ncbi.nlm.nih.gov/subs/bioproject/) under BioProject accession number PRJNA564922.

Received: 12 December 2019; Accepted: 14 April 2020;

Published online: 19 May 2020

\section{References}

1. Mohanty, S. et al. Antibiogram pattern and seasonality of Salmonella serotypes in a North Indian tertiary care hospital. Epidemiol Infect. 134, 961-966, https://doi.org/10.1017/S0950268805005844 (2006).

2. Sharma, P. et al. Changing trends of culture-positive typhoid fever and antimicrobial susceptibility in a tertiary care North Indian Hospital over the last decade. Indian J Med Microbiol. 36, 70-76, https://doi.org/10.4103/ijmm.IJMM-17-412 (2018).

3. Kadhiravan, T. et al. Clinical outcomes in typhoid fever: adverse impact of infection with nalidixic acid-resistant Salmonella typhi. BMC Infect Dis. 5, 37, https://doi.org/10.1186/1471-2334-5-37 (2005).

4. Dahiya, S. et al. Characterisation of antimicrobial resistance in Salmonellae during 2014-2015 from four centres across India: An ICMR antimicrobial resistance surveillance network report. Indian J Med Microbiol. 35, 61-68, https://doi.org/10.4103/ijmm.IJMM16-382 (2017).

5. Kapil, A., Ayyagari, A., Garg, R. K. \& Agarwal, K. C. S. typhi with transferable chloramphenicol resistance isolated in Chandigarh during 1983-87. Indian J Pathol Microbiol. 37, 179-83 (1994).

6. Renuka, K. et al. Reduced susceptibility to ciprofloxacin and gyrA gene mutation in North Indian strains of Salmonella enterica serotype typhi and serotype Paratyphi A. Microb Drug Resist. 10, 146-153 (2004). 
7. Kumar, M. et al. Structure based in silico analysis of quinolone resistance in clinical isolates of Salmonella typhi from India. PLoS One. 10, e0126560, https://doi.org/10.1371/journal.pone.0126560 (2015).

8. Huddleston, J. Horizontal gene transfer in the human gastrointestinal tract: potential spread of antibiotic resistance genes. Infect Drug Resist. 7, 167-76, https://doi.org/10.2147/IDR.S48820 (2014).

9. Gokul, B. N., Menezes, G. A. \& Harish, B. N. ACC-1 beta-lactamase-producing Salmonella enterica serovar Typhi, India. Emerg Infect Dis. 16, 1170-1171, https://doi.org/10.3201/eid1607.091643 (2010).

10. Rodrigues, C. Whole-genome shotgun sequencing of cephalosporin-resistant Salmonella enterica serovar Typhi. Genome Announc. 5(10), e01639-16, https://doi.org/10.1128/genomeA.01639-16 (2017)

11. Hooda, Y. et al. Molecular mechanism of azithromycin resistance among typhoidal Salmonella strains in Bangladesh identified through passive pediatric surveillance. PLoS Negl Trop Dis. 15(13(11)), e0007868, https://doi.org/10.1371/journal.pntd.0007868 (2019).

12. Klemm, E. J. et al. Emergence of an Extensively Drug-Resistant Salmonella enterica Serovar Typhi Clone Harboring a Promiscuous Plasmid Encoding Resistance to Fluoroquinolones and Third-Generation Cephalosporins. MBio. 9 https://doi.org/10.1128/ mBio.00105-18. (2018).

13. Land, M. et al. Insights from 20 years of bacterial genome sequencing. Funct Integr Genomics. 15, 141-161, https://doi.org/10.1007/ s10142-015-0433-4 (2015).

14. Punina, N., Makridakis, N., Remnev, M. \& Topunov, A. Whole-genome sequencing targets drug-resistant bacterial infections. Hum Genomics. 9, 19, https://doi.org/10.1186/s40246-015-0037-z (2015).

15. Bradley, P. et al. Rapid antibiotic-resistance predictions from genome sequence data for Staphylococcus aureus and Mycobacterium tuberculosis. Nat Commun. 6, 10063, https://doi.org/10.1038/ncomms10063 (2015).

16. McDermott, P. F. et al. Whole-Genome Sequencing for Detecting Antimicrobial Resistance in Nontyphoidal Salmonella. Antimicrob Agents Chemother. 60, 5515-5520, https://doi.org/10.1128/AAC.01030-16 (2016).

17. Metcalf, B. J. et al. Using whole genome sequencing to identify resistance determinants and predict antimicrobial resistance phenotypes for year 2015 invasive pneumococcal disease isolates recovered in the United States. Clin Microbiol Infect. 22, 1002. e1-1002.e8, https://doi.org/10.1016/j.cmi.2016.08.001 (2016).

18. Metcalf, B. J. et al. Short-read whole genome sequencing for determination of antimicrobial resistance mechanisms and capsular serotypes of current invasive Streptococcus agalactiae recovered in the USA. Clin Microbiol Infect. 23, 574.e7-574.e14, https://doi. org/10.1016/j.cmi.2017.02.021 (2017).

19. Tyson, G. H. et al. WGS accurately predicts antimicrobial resistance in Escherichia coli. J Antimicrob Chemother. 70, 2763-2769, https://doi.org/10.1093/jac/dkv186 (2015).

20. Zhao, S. et al. Whole-Genome Sequencing Analysis Accurately Predicts Antimicrobial Resistance Phenotypes in Campylobacter spp. Appl Environ Microbiol. 82, 459-466, https://doi.org/10.1128/AEM.02873-15 (2015).

21. Reygaert, W. C. An overview of the antimicrobial resistance mechanisms of bacteria. AIMS Microbiol. 4(3), 482-501, https://doi. org/10.3934/microbiol.2018.3.482 (2018).

22. Britto, C. D., John, J., Verghese, V. P. \& Pollard, A. J. A systematic review of antimicrobial resistance of typhoidal Salmonella in India. The. Indian Journal of Medical Research 149(2), 151-163, https://doi.org/10.4103/ijmr.IJMR_830_18 (2019).

23. Britto, C. D. et al. Persistent circulation of a fluoroquinolone-resistant Salmonella enterica Typhi clone in the Indian subcontinent. The. Journal of Antimicrobial Chemotherapy 75(2), 337-341, https://doi.org/10.1093/jac/dkz4935 (2020).

24. Ruan, Z. \& Feng, Y. BacWGSTdb, a database for genotyping and source tracking bacterial pathogens. Nucleic Acids Res. 44(D1), D682-87. https://doi.org/10.1093/nar/gkv1004.

25. Cao, G. et al. Phylogenetics and Differentiation of Salmonella Newport Lineages by Whole Genome Sequencing. PLoS ONE. 8, e55687, https://doi.org/10.1371/journal.pone.0055687 (2013).

26. Papanikolaou, N. et al. Gene socialization: gene order, GC content and gene silencing in Salmonella. BMC Genomics. 10, 597, https:// doi.org/10.1186/1471-2164-10-597 (2009).

27. Lima, N. C. B. et al. Analysis of isolates from Bangladesh highlights multiple ways to carry resistance genes in Salmonella Typhi. BMC Genomics. 20(1), 530, https://doi.org/10.1186/s12864-019-5916-6 (2019).

28. Fu, S. et al. Defining the Core Genome of Salmonella enterica Serovar Typhimurium for Genomic Surveillance and Epidemiological Typing. J Clin Microbiol. 53, 2530-2538, https://doi.org/10.1128/JCM.03407-14 (2015).

29. Hayden, H. S. et al. Genomic Analysis of Salmonella enterica Serovar Typhimurium Characterizes Strain Diversity for Recent U.S. Salmonellosis Cases and Identifies Mutations Linked to Loss of Fitness under Nitrosative and Oxidative Stress. MBio. 7, e00154, https://doi.org/10.1128/mBio.00154-16 (2016).

30. Mather, A. E. et al. Genomic Analysis of Salmonella enterica Serovar Typhimurium from Wild Passerines in England and Wales. Appl Environ Microbiol. 82, 6728-6735 (2016).

31. Page, A. J. et al. Roary: rapid large-scale prokaryote pan genome analysis. Bioinformatics. 31(22), 3691-3693, https://doi. org/10.1093/bioinformatics/btv421.। (2015).

32. Choudhary, A. et al. Antimicrobial susceptibility of Salmonella enterica serovars in a tertiary care hospital in Southern India. Indian JMed Res. 137, 800-802 (2013).

33. Tettelin, H., Riley, D., Cattuto, C. \& Medini, D. Comparative genomics: the bacterial pan-genome. Curr Opin Microbio. 11, 472-477 (2008).

34. Jacobsen, A. et al. The Salmonella enterica Pan-genome. Microb Ecol. 62, 487-504, https://doi.org/10.1007/s00248-011-9880-1 (2011).

35. Tatusov, R. L., Galperin, M. Y., Natale, D. A. \& Koonin, E. V. The COG database: a tool for genome-scale analysis of protein functions and evolution. Nucleic Acids Res. 28, 33-36, https://doi.org/10.1093/nar/28.1.33 (2000).

36. Kanehisa, M. et al. KEGG as a reference resource for gene and protein annotation. Nucleic Acids Res. 44, D457-D462 2810.1093/nar/ gkv1070 (2016).

37. Katiyar, A., Sharma, S., Singh, T. P. \& Kaur, P. Identification of Shared Molecular Signatures Indicate the Susceptibility of Endometriosis to Multiple Sclerosis. Front Genet. 9, 42, https://doi.org/10.3389/fgene.2018.00042 (2018).

38. Katiyar, A., Singh, H. \& Azad, K.K. Identification of Missing Carbon Fixation Enzymes as Potential Drug Targets in Mycobacterium Tuberculosis. J Integr Bioinform. 15 https://doi.org/10.1515/jib-2017-0041 (2018).

39. Palmer, K. L. et al. Comparative Genomics of Enterococci: Variation in Enterococcus faecalis, Clade Structure in E. faecium, and Defining Characteristics of E. gallinarum and E. casseliflavus. MBio. 3, e00318-11, https://doi.org/10.1128/mBio.00318-11 (2012).

40. Beukers, A. et al. Comparative genomics of Enterococcus spp. isolated from bovine feces. BMC Microbiol. 17, 52, https://doi. org/10.1186/s12866-017-0962-1 (2017).

41. Livermore, D. beta-Lactamases in laboratory and clinical resistance. Clinical Microbiology Reviews. 8, 557-584 (1995).

42. Kang, H. Y. et al. Characterization of antimicrobial resistance and class 1 integrons found in Escherichia coli isolates from humans and animals in Korea. J Antimicrob Chemother. 55, 639-644, https://doi.org/10.1093/jac/dki076 (2005).

43. Martinez-Freijo, P. et al. Many Class I Integrons Comprise Distinct Stable Structures Occurring in Different Species of Enterobacteriaceae Isolated from Widespread Geographic Regions in Europe. Antimicrobial Agents and Chemotherapy. 43, 686-689, https://doi.org/10.1128/AAC.43.3.686 (1999).

44. Balaji, V. et al. Longitudinal Typhoid Fever Trends in India from 2000 to 2015. Am J Trop Med Hyg. 99, 34-40, https://doi. org/10.4269/ajtmh.18-0139 (2018). 
45. Sharma, P. et al. Pefloxacin as a surrogate marker for quinolone susceptibility in Salmonella enterica serovars Typhi \& Paratyphi A in India. Indian J Med Res. 145, 687-692, https://doi.org/10.4103/ijmr.IJMR_494_16 (2017).

46. Sharma, P. et al. Azithromycin resistance mechanisms in typhoidal salmonellae in India: A 25 years analysis. Indian J Med Res. 149, 404-411, https://doi.org/10.4103/ijmr.IJMR_1302_17 (2019).

47. Olliver, A., Valle, M., Chaslus-Dancla, E. \& Cloeckaert, A. Role of an acrR mutation in multidrug resistance of in vitro-selected fluoroquinolone-resistant mutants of Salmonella enterica serovar Typhimurium. FEMS Microbiol Lett. 238, 267-272, https://doi. org/10.1016/j.femsle.2004.07.046 (2004).

48. Nishino, K., Latifi, T. \& Groisman, E. Virulence and drug resistance roles of multidrug efflux systems of Salmonella enterica serovar Typhimurium. Mol Microbiol. 59, 126-141, https://doi.org/10.1111/j.1365-2958.2005.04940.x (2006).

49. Dahiya, S. et al. Multiple locus sequence typing of Salmonella Typhi, isolated in north India - a preliminary study. Indian J Med Res. 137(5), 957-962 (2013)

50. Wong, V. K. et al. An extended genotyping framework for Salmonella enterica serovar Typhi, the cause of human typhoid. Nat Commun. 7, 12827, https://doi.org/10.1038/ncomms12827 (2016).

51. Thanh, D. P. et al. A novel ciprofloxacin-resistant subclade of H58 Salmonella Typhi is associated with fluoroquinolone treatment failure. Elife 5, e14003, https://doi.org/10.7554/eLife.14003 (2016).

52. Britto, C. D. et al. Laboratory and molecular surveillance of paediatric typhoidal Salmonella in Nepal: Antimicrobial resistance and implications for vaccine policy. PLoS neglected tropical diseases. 12(4), e0006408 (2018).

53. Britto, C. D. et al. Persistent circulation of a fluoroquinolone-resistant Salmonella enterica Typhi clone in the Indian subcontinent. Journal of Antimicrobial Chemotherapy. 75(2), 337-341 (2019).

54. Ingle, D. J. et al. Informal genomic surveillance of regional distribution of Salmonella Typhi genotypes and antimicrobial resistance via returning travellers. PLoS Negl Trop Dis. 13(9), e0007620, https://doi.org/10.1371/journal.pntd.0007620 (2019).

55. Wong, V. K. et al. Phylogeographical analysis of the dominant multidrug-resistant H58 clade of Salmonella Typhi identifies inter-and intracontinental transmission events. Nat. Genet. 47, 632-639, https://doi.org/10.1038/ng.3281. (2015).

56. Rahman, S. I. A. Population structure and antimicrobial resistance patterns of Salmonella Typhi isolates in urban Dhaka, Bangladesh from 2004 to 2016. PLoS Negl Trop Dis. 14(2), e0008036, https://doi.org/10.1371/journal.pntd.0008036 (2020).

57. Sharma, P. et al. Use of Multi-Locus Sequence Typing to Determine Population Structure. PLoS One. 11, e0162530, https://doi. org/10.1371/journal.pone.0162530 (2016).

58. StataCorp. Statistical Software: Release 14 College Station, TX: StataCorp LP (2015).

59. Andrews, S. FastQC: a quality control tool for high throughput sequence data Available online at: http://www.bioinformatics. babraham.ac.uk/projects/fastqc (2010).

60. Coil, D., Jospin, G. \& Darling, A. A5-miseq: an updated pipeline to assemble microbial genomes from Illumina MiSeq data. Bioinformatics. 31, 587-589, https://doi.org/10.1093/bioinformatics/btu661 (2014).

61. Lohse, M. et al. RobiNA: a user-friendly, integrated software solution for RNA-Seq-based transcriptomics. Nucleic Acids Res. 40, W622-W627, https://doi.org/10.1093/nar/gks540 (2012).

62. Simpson, J. \& Durbin, R. Efficient de novo assembly of large genomes using compressed data structures. Genome Res. 22, 549-556, https://doi.org/10.1101/gr.126953.111 (2012).

63. Gurevich, A., Saveliev, V., Vyahhi, N. \& Tesler, G. QUAST: quality assessment tool for genome assemblies. Bioinformatics. 29, 1072-1075, https://doi.org/10.1093/bioinformatics/btt086 (2013).

64. Li, W. \& Godzik, A. Cd-hit: a fast program for clustering and comparing large sets of protein or nucleotide sequences. Bioinformatics. 22, 1658-1659 (2006).

65. Seemann, T. Prokka: rapid prokaryotic genome annotation. Bioinformatics. 30, 2068-2069, https://doi.org/10.1093/bioinformatics/ btu153 (2014).

66. Chaudhari, N., Gupta, V. \& Dutta, C. BPGA- an ultra-fast pan-genome analysis pipeline. Sci Rep. 6, 24373. https://doi.org/10.1038/ srep24373 (2016)

67. Edgar, R. Search and clustering orders of magnitude faster than BLAST. Bioinformatics. 26, 2460-2461, https://doi.org/10.1093/ bioinformatics/btq461 (2010).

68. Williams, T. et al. Gnuplot 4.5: An Interactive Plotting Program. URL http://gnuplot.info (2011).

69. Zhao, Y. et al. PanGP: A tool for quickly analyzing bacterial pan-genome profile. Bioinformatics. 30, 1297-1299, https://doi. org/10.1093/bioinformatics/btu017 (2014).

70. Alcock, B.P. et al. CARD 2020: antibiotic resistome surveillance with the comprehensive antibiotic resistance database. Nucleic Acids Res. https://doi.org/10.1093/nar/gkz935 (2019).

71. Hendriksen, R. et al. Using Genomics to Track Global Antimicrobial Resistance. Front Public Health. 7, 242, https://doi.org/10.3389/ fpubh.2019.00242 (2019).

72. Zankari, E. et al. Identification of acquired antimicrobial resistance genes. J Antimicrob Chemother. 67, 2640-2644, https://doi. org/10.1093/jac/dks261 (2012).

73. Clinical and Laboratory Standards Institute (CLSI). Performance standards for antimicrobial susceptibility testing; Twenty-sixth informational supplement. CLSI document M100-S26 Wayne, PA: Clinical and Laboratory Standards Institute (2016).

74. Larsen, M.V., et al., Multilocus Sequence Typing of Total Genome Sequenced Bacteria. J. Clin. Micobiol. 50(4), 1355-1361 https://doi. org/10.12.0/JCM.06094-11 (2012).

75. Alikhan, N., Zhou, Z., Sergeant, M. \& Achtman, M. Escheriachia coli, Salmonella, and Yersinia (EnteroBase): A genomic overview of the population structure of Salmonella website. PLoS Genet. 4, e1.17261 (2018).

76. Kaas, R. S., Leekitcharoenphon, P., Aarestrup, F. M. \& Lund, O. Solving the Problem of Comparing Whole Bacterial Genomes across Different Sequencing Platforms. PLoS ONE 9(8), e104984, https://doi.org/10.1371/journal.pone.0104984 (2014).

77. Rambaut, A. FigTree, version 1.4.3. http://tree.bio.ed.ac.uk/software/figtree/.

78. Letunic, I. \& Bork, P. Interactive Tree Of Life (iTOL) v4: recent updates and new developments. Nucleic Acids Res. 47(W1), W256-W259 (2019).

79. Dyson, Z.A., Klemm, E.J., Palmer, S., \& Dougan, G. Antibiotic Resistance and Typhoid. Clin Infect Dis. 68, S165-S170 https://doi. org/10.1093/cid/ciy1111 (2019).

\section{Acknowledgements}

This work was supported by funding from Indian Council of Medical Research, New Delhi (Grant No. $\mathrm{BIC} / 12(21) / 2012)$.

\section{Author contributions}

P.K., Ar.K. and H.S. contributed to the experimental design. A.K. contributed to W.G.S., genotypic A.M.R. and pan-genome data analysis, interpreted the results and drafted the manuscript. P.S. contributed to phenotypic antimicrobial susceptibility testing, interpreted the results and drafted the manuscript. S.D. was involved in the sample collection and pre-processing. P.K. and Ar.K. reviewed and edited the manuscript. All authors have read and approved the manuscript for publication. 


\section{Competing interests}

The authors declare no competing interests.

\section{Additional information}

Supplementary information is available for this paper at https://doi.org/10.1038/s41598-020-64934-0.

Correspondence and requests for materials should be addressed to A.K. or P.K.

Reprints and permissions information is available at www.nature.com/reprints.

Publisher's note Springer Nature remains neutral with regard to jurisdictional claims in published maps and institutional affiliations.

(c) (i) Open Access This article is licensed under a Creative Commons Attribution 4.0 International License, which permits use, sharing, adaptation, distribution and reproduction in any medium or format, as long as you give appropriate credit to the original author(s) and the source, provide a link to the Creative Commons license, and indicate if changes were made. The images or other third party material in this article are included in the article's Creative Commons license, unless indicated otherwise in a credit line to the material. If material is not included in the article's Creative Commons license and your intended use is not permitted by statutory regulation or exceeds the permitted use, you will need to obtain permission directly from the copyright holder. To view a copy of this license, visit http://creativecommons.org/licenses/by/4.0/.

(c) The Author(s) 2020 